This item was submitted to Loughborough's Research Repository by the author.

Items in Figshare are protected by copyright, with all rights reserved, unless otherwise indicated.

\title{
A technique to predict the aerodynamic effects of battle damage on an aircraft's wing
}

PLEASE CITE THE PUBLISHED VERSION

http://dx.doi.org/10.1017/S0001924000004267

PUBLISHER

(C) Royal Aeronautical Society

VERSION

AM (Accepted Manuscript)

PUBLISHER STATEMENT

This work is made available according to the conditions of the Creative Commons Attribution 4.0 International (CC BY 4.0) licence. Full details of this licence are available at: http://creativecommons.org/licenses/ by/4.0/

\section{LICENCE}

CC BY 4.0

\section{REPOSITORY RECORD}

Pickhaver, T.W., and Peter Render. 2016. "A Technique to Predict the Aerodynamic Effects of Battle Damage on an Aircraft's Wing". Loughborough University. https://hdl.handle.net/2134/17506. 


\title{
A Technique to Predict the Aerodynamic Effects of Battle Damage on an Aircraft's Wing
}

Thomas W. Pickhaver and Peter M. Render

Loughborough University, Loughborough, Leicestershire, LE11 3TU, United Kingdom

\begin{abstract}
A technique is developed that can be used to predict the effects of battle damage on the aerodynamic performance of an aircraft's wing. The technique is based on results obtained from wind tunnel tests on a NASA LS(1)-0417MOD aerofoil with simulated gunfire damage. The wind tunnel model incorporated an internal cavity to represent typical aircraft construction and this was located between $24 \%$ and $75 \%$ of chord. The damage was simulated by circular holes with diameters between $20 \%$ and $40 \%$ of chord. To represent different attack directions, the inclination of the hole axis relative to the aerofoil chord was varied between $\pm 60^{\circ}$ pitch and $45^{\circ}$ of roll. The aerofoil spanned the wind tunnel to create approximate two-dimensional conditions and balance measurements were carried out at a Reynolds number of 500,000 for incidences, increased in $2^{\circ}$ increments, from $-4^{\circ}$ to $16^{\circ}$. Surface flow visualization and pressure measurements were also carried out. For a given hole size, the increments in lift, drag and pitching moment coefficients produced trends when plotted against the difference between the upper and lower surface pressure coefficients on the undamaged aerofoil taken at the location of the damage. These trends are used as the basis of the predictive technique. The technique is used to predict the effects of a previously untested damage case, and these are compared with wind tunnel tests carried out on a half model finite aspect ratio wing. For all coefficients the trends in the predicted data are similar to experiment, although there are some discrepancies in absolute values. For the drag coefficient these discrepancies are partly accounted for by limitations in the technique, whilst discrepancies in the lift and pitching moment coefficients are attributed to limitations in the aerofoil test arrangements.
\end{abstract}

\section{Nomenclature}

$\mathrm{b} \quad=\operatorname{span}$

C $\quad$ chord

$\mathrm{C}_{\mathrm{d}}=$ two dimensional drag coefficient

$\mathrm{C}_{\mathrm{D}} \quad=$ three dimensional drag coefficient

$\mathrm{C}_{\mathrm{l}}=$ two dimensional lift coefficient

$\mathrm{C}_{\mathrm{L}} \quad=$ three dimensional lift coefficient

$\mathrm{C}_{\mathrm{m}} \quad=$ two dimensional pitching moment coefficient 


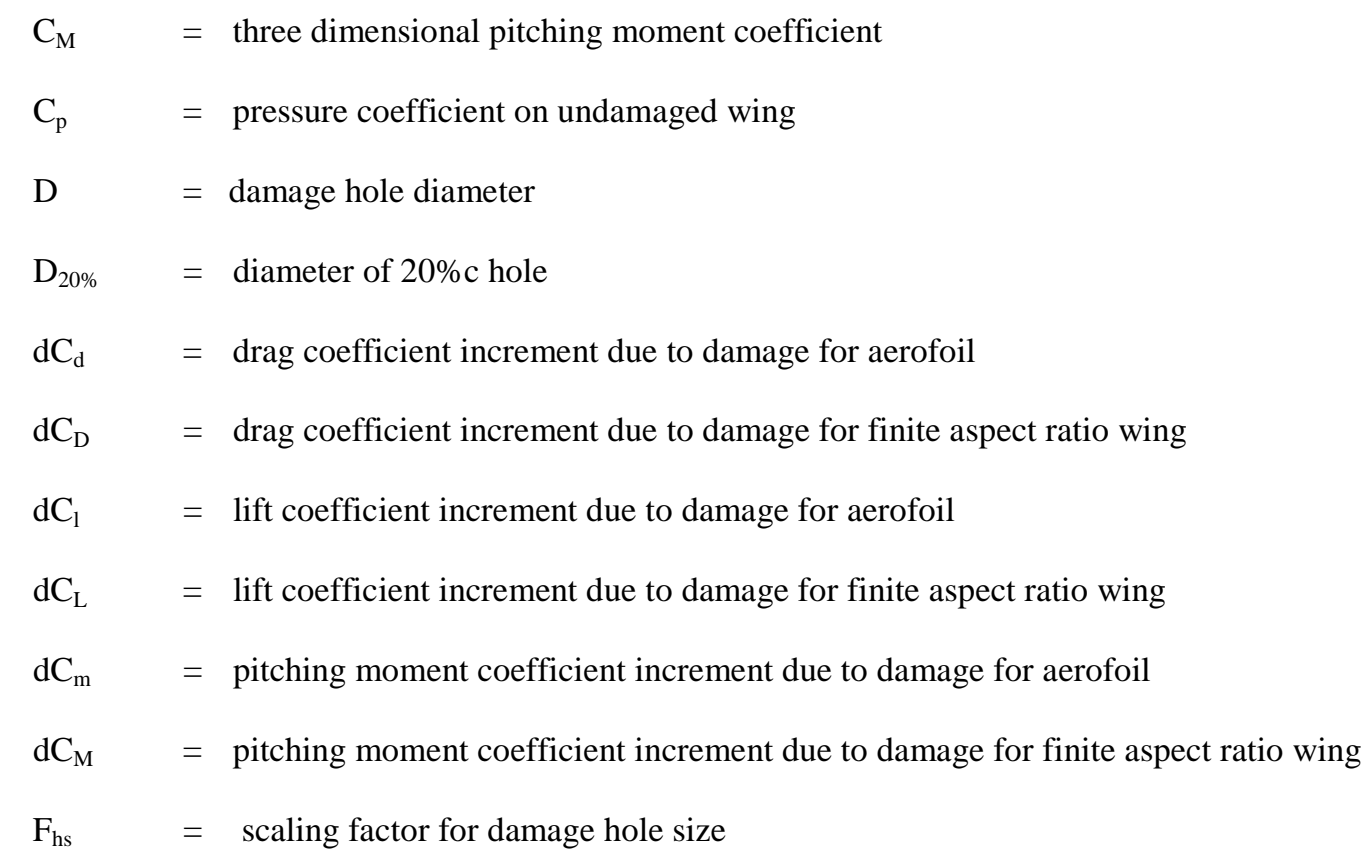

\section{Subscripts}

Dia $\quad=$ diameter of damage hole to be predicted

damaged $\quad=$ results for damaged model

$\mathrm{p} \quad=$ predicted value

Ref $\quad=$ reference hole

undamaged $=$ results for undamaged model

2D $\quad=$ two dimensional

3D $=$ three dimensional

\section{Introduction}

Aircraft survivability in a combat environment is an important aspect of the design process. Most survivability assessments concentrate on structural and systems integrity, but it is known that aircraft can survive a significant level of damage and continue flying. To assess whether an aircraft can survive an attack and still fly to a friendly base requires the ability to estimate the influence of damage on the aerodynamic characteristics of an aircraft. The exact nature of damage sustained by an aircraft is a function of many variables (e.g. weapon type, attack angle, etc.) so any method used to determine the aerodynamic effects of damage must be able to consider a wide range of 
damage scenarios. Ideally the method should also be straight forward to use so that critical damage scenarios can be quickly identified, and then analysed in more detail.

The main aim of the present study is to develop a simple technique to predict the effects of battle damage on the aerodynamic characteristics of a finite aspect ratio wing. Such a method will allow the survivability analyst to identify critical damage cases which may require more detailed investigation by either numerical or experimental methods. Whilst computational methods ${ }^{1,2}$ can be used to predict the effects of battle damage with a reasonable degree of accuracy, the time required to generate model grids makes them unsuitable for rapidly assessing the wide range of possible damage scenarios on a wing in a short period of time. Possible damage cases on other lifting surfaces (e.g. fin and tailplane) add to the complexity of the survivability analyst's task and increases the desirability of having a simple predictive technique.

Whilst developing the predictive technique, the paper also extends the existing experimental knowledge base on battle damage by investigating the aerodynamic effects of damage arising from a range of attack directions.

\section{Previous Studies}

The first systematic investigation into the aerodynamic effects of battle damage was carried out by Irwin and Render $^{3}$. In their paper they noted that "previous work had considered the effects of very simple forms of damage on aircraft models at high speeds, while examinations into low speed characteristics have failed to explain the aerodynamic effects”. Irwin and Render ${ }^{3}$ investigated the effect of simulated gun fire damage on the twodimensional characteristics of a NACA $64_{1}-412$ aerofoil. The damage was modelled by circular holes located at either quarter or half chord with diameters ranging from $10 \%$ to $40 \%$ of aerofoil chord. The flow through the damage hole was shown to have many of the features identified by Andreopoulos and Rodi ${ }^{4}$ for a jet in cross flow when it emerges from a flat plate. This allowed the flow through the damage to be characterised as being either a weak or strong jet. A sketch of the key features of a weak jet is reproduced from Irwin and Render ${ }^{3}$ and shown as Fig. 1. Forward of the damage hole, the flow over the wing separated at the forward separation line and a horseshoe vortex was formed. Upon exit, the jet through the hole was immediately bent over and attached itself to the surface of the wing. At the downstream edge of the hole a contra rotating vortex pair was seen. This vortex pair is a significant feature of jets in cross flow and as described by Mahesh ${ }^{5}$ different mechanisms have been proposed to 
explain their production. Downstream of the hole, the flow in the attached wake was seen to have a varying velocity profile with the highest values at the edges. Irwin and Render ${ }^{3}$ determined that weak jets resulted in small changes in lift, drag and pitching moment, and were associated with small holes or larger holes at low incidence. Irwin and Render's ${ }^{3}$ sketch of a strong jet is shown in Fig.2. A forward separation line indicating the formation of a horseshoe vortex was again seen upstream of the damage. Behind the damage the spanwise distance between the two arms of the horseshoe vortex was far greater than seen for the weak jet. Towards the trailing edge, the horseshoe vortex ended in a pair of contra rotating vortices on the surface of the wing, with reverse flow between them which was entrained around the trailing edge of the wing. As this reverse flow approached the damage it was entrained into the strong jet which had exited through the damage hole and penetrated into the freestream flow. It was observed that strong jets resulted in large changes in aerodynamic forces and moments, and were associated with large diameter holes and small holes at high incidence.

Irwin and Render ${ }^{6}$ investigated the influence of a wing's internal structure and showed that the presence of a cavity reduced the size of lift and drag changes due to damage. Although these changes were relatively small, they became more significant as incidence or hole size increased, which indicates that modelling of the internal structure is desirable for battle damage studies.

Two criticisms that can be levelled against Irwin and Render $^{3}$ are that circular holes are unrepresentative of battle damage and that they ignored petalling which often takes place around a hole when a projectile passes through a metal structure. Render et al. ${ }^{7}$ investigated the effect of hole shape by considering star shaped damage. Despite the complexity of the flow through the damage, flow visualisation revealed that the flow could still be categorised as a weak or strong jet with features broadly similar to those identified by Irwin and Render ${ }^{3}$. By considering both flow visualisation and the size of lift, drag and pitching moment changes due to damage it was concluded that circular holes are a reasonable representation of battle damage, provided the diameter is close to the maximum width of the damage being simulated. These findings are broadly in line with those of Robinson and Leishman ${ }^{8}$ who investigated ballistic effects on helicopter rotor blades and concluded that the shape of the damage played only a minor role in the aerodynamic degradation. Robinson and Leishman ${ }^{8}$ also used serrations to simulate petalling, but these did not change the basic characteristics of the flow through the damage, although the resulting wake appeared to be more 
energetic. In terms of the aerodynamic losses it was concluded that the hole rather than the serrations was the dominant source of changes in aerodynamic forces and moments.

Computational studies using a circular hole with a diameter of $30 \%$ of aerofoil chord and a star shaped case from Render $^{7}$ have been carried out by Saeedi et al. ${ }^{1}$ In both cases the wing was solid and there was no attempt to model an internal wing geometry. The numerical simulations confirmed the flow features seen in the wind tunnel for both weak and strong jets, and the predicted effects of the damage on lift, drag and pitching moment were close to experiments, although the agreement diverged as incidence was increased towards stall. Saeedi et al. ${ }^{1}$ also investigated the flow inside the hole and observed a complicated vortex arrangement of interacting vortices in the upper and lower parts of the hole, which were also symmetrically placed on the left and right sides of the hole.

The aerodynamic effects of battle damage on finite aspect ratio wings was investigated by Render et al. ${ }^{9}$ for constant chord unswept wings between aspect ratios of 6 and 10. The flow through the damage was asymmetric, i.e.: differences existed between the outboard and inboard sides of the damage. This was attributed to the variation in static pressure along the span of the wing which weakened the jet slightly on the outboard side. However, the jet still retained the flow characteristics observed by Irwin and Render $^{3}$ and also seen for jets in cross flow. Render et al. ${ }^{9}$ also showed that the effects of damage on lift and drag can be related to the difference between the pressure coefficients on the upper and lower surfaces of the undamaged wing at the location of the damage. This gives rise to the possibility of using the pressure distribution around an undamaged wing to predict the likely effects of damage.

Most recently Render and Pickhaver ${ }^{10}$ have investigated the effects of battle damage on a NASA LS aerofoil and investigated the effects of attack direction. Changes in attack direction result in the upper and lower surface holes being displaced relative to each other. This initial investigation into attack direction is extended in the current paper. Pickhaver and Render ${ }^{11}$ then demonstrated that the techniques developed by Render et al. ${ }^{9}$ could be successfully used to predict the effects of damage on a wing with an aspect ratio of 6 . A significant finding identified by Pickhaver and Render ${ }^{11}$ was that for all attack directions, the lift, drag and pitching moment increments were related to the difference between the pressure coefficients on the upper and lower surfaces of the undamaged wing at the location of the damage. When plotting increment data against difference in pressure coefficient, it was seen that 
there was no distinction between weak and strong jets. Pickhaver and Render ${ }^{11}$ also took the opportunity to investigate the effects of Reynolds number and concluded that between Reynolds numbers of $10^{6}$ and $5 \times 10^{5}$ there was no noticeable difference in the aerodynamic effects of battle damage.

\section{Aims and Objectives}

As previously stated, the main aim of the present study is to develop a simple technique to predict the effects of battle damage on the aerodynamic characteristics of a finite aspect ratio wing. Render et al. ${ }^{9}$ provide the basis of a predictive technique, but there remains a significant drawback with the proposed method because it requires input data on the aerodynamic effects for each damage geometry of interest. This data could be obtained by either wind tunnel testing or computational methods, but for a large number of damage cases this would involve significant time and effort. Such an expenditure of time and effort is undesirable at the early stages of a survivability analysis, where the emphasis is on quickly identifying critical damage conditions for detailed analysis. This paper demonstrates that the use of a limited input data set (e.g. 2 damage cases) can be used to predict a range of damage conditions.

Nearly all of the previously described battle damage studies have considered simulated gunfire damage that was normal to the aerofoil's chord line. In other words, the wing was hit by a shell or bullet which was fired from either directly below or directly above. In reality gun fire can come from a range of attack angles. For example an attack direction of ahead and below an aircraft is typical of anti-aircraft gun emplacements, whilst attacks from above and behind are typical of cannon fire from enemy aircraft. This paper investigates the effects of attack direction and incorporates the findings into the developed predictive technique.

The stages used to develop the predictive technique were:

1) Wind tunnel tests on a two-dimensional aerofoil model to provide input data for the predictive technique. This included determining the aerodynamic effects of damage for a range of hole diameters and attack angles.

2) Wind tunnel tests on a finite aspect ratio wing. This served two purposes:

a. To obtain pressure coefficient data for an undamaged wing. These results were used as an input to the predictive technique. 
b. To determine the aerodynamic effects of some of the damage cases previously tested on the twodimensional aerofoil. This provided data for both developing the predictive technique and assessing the accuracy of predicted results.

3) Use of the predictive technique to predict the effects of previously untested damage cases on the aerodynamic characteristics of a finite aspect ratio wing.

4) Wind tunnel testing of the finite aspect ratio wing with the previously untested damage cases.

5) Comparison of predicted results from 3) with wind tunnel tests from 4) to assess the accuracy of the predictive technique.

The NASA LS(1)-0417MOD aerofoil ${ }^{12}$ was selected for the study since it is a more modern design than the NACA aerofoil used by Irwin and Render ${ }^{3}$. The aerofoil is also likely to be similar to those found on modern low speed aircraft such as Unmanned Air Vehicles (UAV) designed for the reconnaissance or surveillance roles.

\section{Two-Dimensional Test Arrangement}

The aerofoil model had a chord of $200 \mathrm{~mm}$ and incorporated an internal cavity between $0.24 \mathrm{c}$ and $0.75 \mathrm{c}$ to replicate the internal structure of an aircraft wing (Fig. 3). The leading and trailing edges of the model were solid and manufactured from ProLab 65 which is a synthetic modelling board. The top and bottom of the cavity were formed by removable panels. These panels were moulded from fibreglass and attached to the model by countersunk screws. The aerofoil model was installed in Loughborough University's Low Turbulence Wind Tunnel which has a working section $0.45 \mathrm{~m} \times 0.45 \mathrm{~m}$ and a turbulence intensity of typically $0.1 \%$. The model was mounted on to a balance beneath the working section by means of fore and aft struts, and spanned the working section to give approximately two-dimensional conditions for the undamaged aerofoil (Fig. 4). Incidence was increased from $-4^{\circ}$ to $15^{\circ}$ which covered the zero-lift and stall of the aerofoil. Increments in incidence were $2^{\circ}$, reducing to $1^{\circ}$ as stall was approached. The balance measured lift, drag and pitching moment with calibrated accuracies of better than $0.05 \%$ full scale deflection. Balance readings were recorded by a PC using LabView software and a National Instruments CompactRIO data acquisition system. A mixture of titanium dioxide, paraffin and linseed oil was used to obtain flow visualisation at the same incidences used for balance measurements. All tests were run at an air velocity of around $37 \mathrm{~m} / \mathrm{s}$, which was close to the wind tunnel's maximum, and resulted in a Reynolds Number of 500,000. A transition strip was installed on the upper surface at $0.075 \mathrm{c}$ to minimize potential Reynolds number effects. 
Simulated battle damage consisting of circular holes was added to the removable panels, with a new set of panels used for each damage case tested. As reported by Irwin and Render ${ }^{3}$ gunfire damage can result in hole diameters ranging from 5\% of local wing chord (denoted 5\%c) for a small shell at the wing root to $100 \%$ c for a large antiaircraft shell close to a wing tip. To retain structural integrity of the removable wing panels, three hole diameters of 20\%c, 30\%c and 40\%c are considered in this paper. Diameters of 5\%c and 10\%c were also tested during the study, but changes in forces and moments were small and difficult to distinguish from repeatability errors. The angular orientation of the damage was:

a) Straight through with the holes on the upper and lower surface immediately above each other.

b) Positive and negative obliquity with the upper and lower surface holes displaced along the chord. (i.e.: the axis of the line joining the hole centres passed through the half chord location on the aerofoil's chord, but was rotated fore and aft.) Negative obliquity resulted in the upper surface hole being closer to the aerofoil's leading edge.

c) Skew with the upper and lower surface holes displaced along the span of the model. (i.e.: the axis of the line joining the hole centres passed through the chord at the model's mid span location but was rotated towards the model's tips.) Given the symmetry of two-dimensional testing there was no need to define a sign convention, but in this paper skew was achieved by moving the upper surface hole to the left and the lower hole to the right.

d) Combined skew and obliquity.

Figure 5 illustrates the definition of obliquity for $+60^{\circ}$. The black regions indicate the hole and the hatched regions indicate the solid leading and trailing edges. The dashed line indicates the chord line and the dotted line indicates the obliquity angle, centred on the chord line. The combinations of damage hole size and angular orientation considered, in this paper, although not necessarily discussed, are summarised in Table 1.

The length of the model's chord was chosen to allow more precise damage modelling. However, this gave a chord to tunnel height ratio of 0.444 , which is significantly larger than the normally accepted maximum of $0.35^{13}$. Wind tunnel corrections were applied to balance measurements using the method of Garner ${ }^{14}$. The applicability of this method was established at the start of the study when wind tunnel tests were carried out on undamaged models of 200 and $141 \mathrm{~mm}$ at the same Reynolds number. The latter model gave a chord to tunnel height ratio of 0.3 . 
Comparing the results from the two models indicated that the adopted wind tunnel corrections were valid for the 200 mm chord model.

The acceptability of the data from the undamaged model was assessed by comparing with NASA wind tunnel data $^{12}$ collected at a Reynolds number of 2,000,000. This was the lowest Reynolds number at which data was available, and a comparison was only possible because of the presence of a transition strip on the undamaged model. Unlike the present study, the NASA data for lift and pitching moment coefficients was obtained from surface pressure measurements and the drag data from a wake survey and this will account for some of the discrepancies between the two sets of data shown in Fig. 6. However, the main cause of discrepancies can be attributed to the difference in model construction. The NASA data is for an aerofoil model with a smooth continuous and accurate contour which was not the case for the undamaged hollow model with its removable panels. Despite fitting well, the panels produced discontinuities in the model surface. Flow visualisation showed that compared to the rest of the model the upper surface panel produced early separation over the rear of the model and resulted in a premature stall (Fig. 6a). This flow separation combined with the surface discontinuities resulted in increased drag coefficient across the incidence range (Fig. 6b). The pitching moment coefficients for the two models (Fig. 6c) were similar, although the present study produced smaller values. This is believed to be due to friction in the pin joints used for mounting the present model in the wind tunnel. Taking into account the differences in model profiles, comparisons with the NASA data indicated that acceptable undamaged data was produced by the present study.

One set of undamaged panels were pressure tapped to provide surface pressure measurements. Chordwise tappings were placed on the centreline of the panel at fixed intervals of $10 \mathrm{~mm}$. The tappings were connected to a Pressure Systems 16TC/DTC pressure scanner linked to a PC via a Chell CanDAQ data acquisition unit. The data acquisition software sampled each pressure tapping 8,192 times over a period of approximately 30 seconds. The nominal accuracy of the pressure measurements was $\pm 0.0696 \mathrm{mmH}_{2} \mathrm{O}$.

The results for the damage cases are presented as increments in lift, drag and pitching moment coefficients for the undamaged wing and are defined as:

$$
\begin{gathered}
\mathrm{dC}_{\mathrm{l}}=\mathrm{C}_{\mathrm{l} \text { damaged }}-\mathrm{C}_{\mathrm{l} \text { undamaged }} \\
\mathrm{dC}_{\mathrm{d}}=\mathrm{C}_{\mathrm{d} \text { damaged }}-\mathrm{C}_{\mathrm{d} \text { undamaged }} \\
\mathrm{dC}_{m}=\mathrm{C}_{\mathrm{m} \text { damaged }}-\mathrm{C}_{\mathrm{m} \text { undamaged }}
\end{gathered}
$$


where lower case $\mathrm{l}, \mathrm{d}$ and $\mathrm{m}$ denote two-dimensional wing quantities. Tests on different damage cases indicated that the repeatability levels for the increments were \pm 0.013 for $\mathrm{dC}_{\mathrm{l}}, \pm 0.0013$ for $\mathrm{dC}_{\mathrm{d}}$ and \pm 0.0020 for $\mathrm{dC}_{\mathrm{m}}$.

\section{The Influence of Attack Direction}

Flow visualisation for $20 \%$ c straight through damage is shown in Fig. 7 . The leading edge of the model is at the bottom of the pictures. To avoid contamination of the transition strip the flow visualization mixture was applied downstream of the strip. Using the key flow features identified by Irwin and Render ${ }^{3}$ a qualitative assessment of jet strength can be made. Figure 7a is at zero incidence and shows the sides of a horseshoe vortex (A) which formed around the front of the hole and then travelled to the trailing edge of the wing. Behind the hole, and bounded by the sides of the horseshoe vortex, was an attached wake (B). The horseshoe vortex and the attached wake are the key features of a weak jet identified by Irwin and Render ${ }^{3}$. The flow through the damage strengthened with increasing incidence, and as shown in Fig. 7b, at $4^{\circ}$ all of the features described by Irwin and Render ${ }^{3}$ for a strong jet were present. The presence of a horseshoe vortex is indicated by the forward separation line (A) and the contra rotating vortex pair (B) on the downstream edge of the damage hole are both characteristics of jets in cross flows. The sides of the horseshoe vortex terminated in two large contra rotating vortices $(\mathrm{C})$ on the surface of the model and between them was a region of reverse flow (D) that was entrained around the trailing edge of the model. This flow moved towards the hole until it met the damage jet expanding downstream of the hole. At this point the reverse flow separated from the model's surface and was entrained into damage jet. Increasing incidence to $8^{\circ}$ resulted in further strengthening of the damage jet and an increased size of spanwise disturbance to the flow over the upper surface of the model (Fig. 7c).

Introducing obliquity had a marked effect on the flow through the damage as is shown in Fig. 8. The two pictures are for $20 \%$ c damage at $+60^{\circ}$ and $-60^{\circ}$ obliquity and can be compared with the straight through case shown in Fig. 7c. The three pictures illustrate how the jet strength and the extent of the flow disturbance increased as the obliquity became more negative. Although not shown, the results for $+30^{\circ}$ and $-30^{\circ}$ obliquity fitted in with these trends. The increasing jet strength was evident at all incidences and can be attributed to the increased pressure difference across the damage hole as obliquity was increased. The obliquity cases are still effectively jets in cross flow, but due to the presence of the internal cavity they are not similar to the inclined jets in cross flow studied by workers such as 
Compton and Johnston ${ }^{15}$ and Milanovic and Zaman ${ }^{16}$. In these studies the axis of the jet at exit was specifically inclined to the freestream flow. To investigate the effects of the cavity, flow visualisation studies were carried out on the inside of the removable panels. When viewing the internal flow visualisation pictures it should be noted that the panels had to be removed from the model to allow the photographs to be taken. As a result flow visualisation mixture sometimes flowed whilst the panels were removed. The pairs of photographs in Figs. 9 to 11 are of the upper and lower panels for one damage case at $8^{\circ}$ incidence. The leading edge of each panel is at the bottom of the photograph, and the top and bottom of each photograph coincide with the start of the wing spars. For the $-60^{\circ}$ obliquity case there is little evidence of flow over the lower panel (Fig. 9a) apart from two inclined lines (A) either side of the hole and a small collection of liquid on either side and towards the rear of the hole (B). By contrast the upper surface (Fig. 9b) shows significant flow as is evident from the relative absence of mixture in the central part of the panel. The mixture at B is a contra rotating vortex pair and indicates the presence of jet flow through the lower hole. The jet impinges on the upper surface at $\mathrm{C}$ and spreads sideways and then flows along the upper surface and exits through the rear of the upper hole. The inclined lines (A) are separation lines and show that there is some flow along the central part of the lower surface with air becoming entrained into the flow through the upper hole. Apart from spreading out along the rear spar, the flow is limited to a narrow part of the cavity and there is no appreciable flow over outboard parts.

The internal flow visualisation for the straight through case is shown in Fig. 10 and it is clear from the upper surface panel (Fig. 10b) that virtually all of the internal flow took place in the rear half of the cavity. Good quality photographs for the lower surface proved difficult to get because a pool of mixture collected towards the rear of the hole and tended to run whilst the panel was removed. From the lower surface panel (Fig. 10a) it appears that upon entering the cavity at A, the air flowed rearwards, spread along the rear spar (B) and then towards the upper panel where it exited through the rear of the upper surface hole (i.e. behind C in Fig. 10b). This flow behaviour within the cavity is similar to that seen for $-60^{\circ}$ obliquity. Interestingly the internal flow visualisation suggests that the jet exited from the rear of the hole. This is in contrast to Fig. 7c which by the position of the horseshoe vortex indicates that the jet occupied the entire hole. This apparent contradiction suggests that some flow passed through the front of the lower and upper holes without entering the cavity. At $+60^{\circ}$ obliquity (Fig. 11) there was less evidence of flow along the lower panel towards the rear spar. The collection of liquid behind the hole (A in Fig. 11a) and the bulge in the flow pattern forward of the hole on the upper surface (B in Fig. 11b) suggests that upon entering the cavity the 
flow through the lower hole had sufficient momentum to impact on the upper surface and then spread out to the sides before exiting through the upper surface hole. It should be noted that due to the presence of the rear spar there was little flow through the most rearward part of the upper surface hole.

Based on the external flow visualisation it was anticipated that reducing the obliquity from $+60^{\circ}$ would result in a reduction in lift coefficient and an increase in drag coefficient at any given incidence. This is confirmed by the coefficient increments shown in Fig. 12. For all increments there were broadly three regions. At the lowest incidences the damage flow for all cases was a weak jet and the increments were similar for all obliquity angles. The onset of strong jet flow marked the start of the second region. For the $-60^{\circ}$ obliquity case the transition to strong jet flow occurred at around $0^{\circ}$ of incidence whilst this was delayed to $4^{\circ}$ for $+60^{\circ}$ obliquity. With the strong jet established, the increments became larger and a distinct trend developed with obliquity For all increments the effects of damage reduced after $10^{\circ}$ of incidence and the trends with obliquity were less well defined. This represents the third region and coincides with the upper limit of the linear portion of the undamaged lift curve slope and the onset of significant separation over the rear of the undamaged model behind the panel.

The behaviour of the $+60^{\circ}$ obliquity case is noteworthy as it was not always consistent with the other cases. Both the lift coefficient (Fig. 12a) and the drag coefficient (Fig. 12b) increments peaked at $6^{\circ}$ incidence as opposed to $10^{\circ}$ for all of the other obliquity cases. For the pitching moment coefficient increments (Fig. 12c) the effects were more subtle, but it can be observed that the $+60^{\circ}$ obliquity case crossed the $+30^{\circ}$ curve at $8^{\circ}$ incidence. The behaviour of the $+60^{\circ}$ obliquity case can be explained by flow visualization on the upper surface. At $4^{\circ}$ (Fig. 13a) the damage flow was a strong jet. At $6^{0}$ (Fig. 13b) the jet appeared to weaken since the extent of the wake was visibly smaller. The jet remained in this state for further increases in incidence (Fig. 8a). From Fig. 11 it is known that at these higher incidences the flow through the lower hole impacted on the underside of the upper surface panel before exiting through the hole. However the flow was distinctly different at lower incidences. Figure 14 shows the internal flow visualisation for $+60^{\circ}$ obliquity at an incidence of $4^{\circ}$. The arrangement of the photographs is the same as used in Fig. 11. There is distinct flow on the lower surface with the flow features of a contra rotating vortex pair (A in Fig. 14a) and an attached wake (B) indicating a weak jet. Upon reaching the rear spar the flow spreads sideways (C) and up towards the upper surface and exits through the rear of the hole, although the presence of the spar prevents flow at the furthest aft point of the hole. The flow through the bottom hole has changed its behaviour by $8^{\circ}$ incidence 
(Fig. 11) to impact on the upper surface. It is suggested that this change in behaviour and loss of momentum is responsible for the damage jet on the upper surface losing its strength.

Skewing the damage hole brought about little change in the increments previously shown for the straight through and obliquity cases. This is not surprising since the pressure difference across the damage hole is unlikely to change significantly with skew as there is no spanwise variation in the surface pressures of an undamaged two dimensional wing. However, skew did introduce asymmetry into the flow at all incidences. This is illustrated by Fig. 15 for a skew angle of $60^{\circ}$ at an incidence of $8^{\circ}$. Comparison with the straight through case in Fig. 7c shows jets of comparable strengths, but the skew case is asymmetric. This is most clearly shown by the contra rotating vortex pair on the edge of the hole which appear to be slightly asymmetric. This asymmetry is introduced by internal flows within the cavity. Flow visualization on the inside surfaces of the cavity (Fig. 16) showed the flow entering through the lower hole impacted on the upper surface of the cavity (A in Fig. 16b ) and then spread out before exiting the hole between the positions B in Fig. 16b.

\section{Development of the Predictive Technique}

Render et al. ${ }^{9}$ showed that for straight through damage the value of a coefficient increment was related to the difference between the pressure coefficients on the upper and lower surfaces of the undamaged wing at the damage location $\left(\mathrm{dC}_{\mathrm{p}}\right)$. Figure 17 shows the pre-stall coefficient increments for the $20 \%$ c hole cases with and without obliquity, including both weak and strong jets, plotted against $\mathrm{dC}_{\mathrm{p}}$ for the undamaged aerofoil. The values of $\mathrm{dC}_{\mathrm{p}}$ were obtained by integrating surface pressures obtained from wind tunnel measurements over the areas of the holes at the upper and lower locations. In Fig. 17 a trend for each increment with $\mathrm{dC}_{\mathrm{p}}$ is apparent, admittedly with some scatter. However it is important to note that the scatter is not due to trends in the data resulting from changes in obliquity or skew. Best fit curves could be placed through each data set and these curves could be used to estimate the likely effects of a $20 \%$ c hole at any combination of skew and obliquity between the tested extremes of $\pm 60^{\circ}$ obliquity.

The data in Fig. 17 has been compiled using results from six different obliquity cases. This represents a significant amount of wind tunnel testing and is contrary to the idea of producing a predictive technique that requires minimal input data. A realistic minimal input data set could comprise of the extreme damage cases of interest, which in this study would be $+60^{\circ}$ and $-60^{\circ}$ obliquity. This would reduce the number of wind tunnel test cases to two. These two 
cases are highlighted in Fig. 17 along with the best fit curves through this data. The curves were derived from a second order least squares fit, which given the scatter in the measured data, were deemed to give an acceptable level of accuracy. For all coefficient increments the best fit curves are close to the curves through all of the data and indicate that the use of the two extreme damage cases will provide an acceptable alternative for estimating the effects of $20 \%$ c damage holes. For reference the equations of the best fit curves through the two extreme damage cases are reproduced below since they are used in the following analysis.

$$
\begin{array}{r}
d C_{l p}=-0.785237\left(d C_{p}\right)^{2}+0.0679725\left(d C_{p}\right) \\
d C_{d p}=0.0376766\left(d C_{p}\right)^{2}+3.87867 \times 10^{-3}\left(d C_{p}\right) \\
d C_{m p}=-0.0450634\left(d C_{p}\right)^{2}-0.0180391\left(d C_{p}\right)
\end{array}
$$

where $\mathrm{dC}_{\mathrm{lp}}, \mathrm{dC}_{\mathrm{dp}}$ and $\mathrm{dC}_{\mathrm{mp}}$ are the predicted coefficient increments for $20 \%$ c damage on the LS(1)-417MOD aerofoil.

The damage cases of interest are unlikely to be just one diameter, so it is necessary to consider the effects of damage size. This is illustrated by Fig. 18 where the increments for straight through cases of three different diameters (20\%c, 30\%c and 40\%c) have been plotted against $\mathrm{dC}_{\mathrm{p}}$. Increasing the hole diameter resulted in increased jet strength and larger increments which is consistent with the findings of Irwin and Render ${ }^{3}$. As with Irwin and Render, the present study found that the pitching moment increments (Fig. 18c) were largely independent of damage size. This suggests that the pitching moment results for the $20 \%$ c case could be applied to any hole diameter up to 40\%c. For the lift and drag increments it would be possible to produce best fits curves for a range of hole sizes and then interpolate to intermediate diameters of interest. However, the aim of the predictive technique is to produce a method that requires only limited inputs from wind tunnel testing or numerical analysis, and the testing or computational analysis to produce best fir curves for a range of hole sizes is likely to be significant. Shown in Figs. 18a and $\mathrm{b}$ are least squares straight lines for the data from each diameter. Normalising the gradients of the lines for the two largest holes by the gradient of the $20 \%$ c line, produces the values in Table 2 . Also in Table 2 are the hole diameters normalised by the diameter of the $20 \%$ c hole. The value of the normalised hole diameter is within $10 \%$ of the normalised line gradients and it is suggested that over a limited range of hole sizes the use of diameter ratio is a convenient method to account for the effects of hole diameter. This can be expressed as:

$$
d C_{l p \text { Dia }}=F_{h s} d C_{l p}
$$




$$
d C_{d p \text { Dia }}=F_{h s} d C_{d p}
$$

where $d C_{l p \text { Dia }}$ and $d C_{d p \text { Dia }}$ denote the increment for the damage size of interest, $d C_{l p}$ and $d C_{d p}$ are values obtained from equations 4 and $5 . \mathrm{F}_{\mathrm{hs}}$ is a is the normalised diameter defined by:

$$
F_{h s}=\frac{D_{D i a}}{D_{R e f}}
$$

where $D_{\text {Dia }}$ is the diameter of the damage of interest and $D_{\text {Ref }}$ is the diameter of the reference hole used to formulate the best fit curves (equations 4 to 6). For the present study $\mathrm{D}_{\text {Ref }}$ is $20 \%$ c.

Analysis of the data collected by Irwin ${ }^{17}$ produced similar results to those shown in Table 2, and it is believed that the use of $F_{h s}$ is likely to be applicable to all aerofoils provided that $1<\mathrm{F}_{\mathrm{hs}}<2$. Whist this method is approximate, its advantage lies in that no additional wind tunnel testing or computational runs are required to account for hole size. For smaller holes the use of $\mathrm{F}_{\mathrm{hs}}$ is likely to remain appropriate. However it is important to note that small holes produce weak jet flows over large parts of the incidence range, and consequently small coefficient increments. Use of the $20 \%$ c hole which had a strong jet by $4^{\circ}$ incidence means that the outlined method is essentially a strong jet method and is likely to over predict the magnitude of increments for small holes.

The method outlined so far produces predictions of the coefficient increments for an aerofoil with battle damage with skew and obliquity. To analyse an aircraft, these increments will need to be converted to the geometry of the finite aspect ratio wing. The basic method has been outlined by Render ${ }^{9}$, but the method was developed for a special case where the chords of the aerofoil and the finite aspect ratio wing were identical. The method has been extended by Pickhaver ${ }^{18}$ to allow predictions for a finite aspect ratio wing with sweep and taper, but for the present paper an unswept and untapered wing is considered. The equations for this type of wing are:

$$
\begin{aligned}
& d C_{L}=d C_{l}\left(\frac{b_{2 D}}{c_{2 D}}\right)\left(\frac{c_{3 D}}{b_{3 D}}\right) \\
& d C_{D}=d C_{d}\left(\frac{b_{2 D}}{c_{2 D}}\right)\left(\frac{c_{3 D}}{b_{3 D}}\right) \\
& d C_{M}=d C_{m}\left(\frac{b_{2 D}}{c_{2 D}}\right)\left(\frac{c_{3 D}}{b_{3 D}}\right)
\end{aligned}
$$

The use of upper case subscripts indicate that $d C_{L}, d C_{D}$ and $d C_{M}$ are the lift, drag and pitching moment coefficient increments for the finite aspect ratio wing. In addition, $b$ is wing span and $\mathrm{c}$ is wing chord with the subscripts 2D and $3 \mathrm{D}$ being used to identify the two dimensional and three dimensional wings respectively. Using the predicted 
coefficient increment values from equations 6,7 and 8 in equations 10 to 12 yield the final predicted coefficient increments for the finite aspect ratio wing.

\section{Assessment of Results from the Predictive Technique}

During its development, the predictive technique was assessed against battle damage wind tunnel data, collected during the present study. However a more realistic assessment is to mimic the future use of the technique, by predicting previously untested damage cases. Two damage cases were defined and tested on an existing finite aspect ratio half wing model. The model had been previously used for battle damage studies and had the same aerofoil and internal construction as the model used in the previously described two dimensional tests. The cavity extended from the wing root to $85 \%$ of span, with the solid tip required to maintain the strength and stiffness of the model. The removable panels were located at three different spanwise locations, so that the centres of the panels were located at 25\%, 50\% and 75\% of span. The model was untwisted and had a constant chord of $325 \mathrm{~mm}$ and a span of $975 \mathrm{~mm}$. The half model configuration is shown in Fig. 19 and gave an effective aspect ratio of 6. Tests were carried out in the Loughborough University $1.9 \mathrm{~m}$ x 1.3 m closed working section wind tunnel, which had a turbulence intensity of less than $0.15 \%$, at the model Reynolds number of $1 \times 10^{6}$. This was a higher Reynolds number than could be achieved for two-dimensional testing, but testing at the higher Reynolds number was desirable to minimise

inaccuracies in test data. Both the two and three dimensional models had transition strips at the same locations to minimise the effects of Reynolds number, but finite aspect ratio tests were also conducted at $5 \mathrm{x} 10^{5}$ to ensure that there were no significant Reynolds number effects on the coefficient increments presented in Figs. 20 and 21.

The experimental coefficient increments for the finite aspect ratio wing are defined by equations to 1 to 3 , but use upper case letters as subscripts to identify three dimensional coefficients with the resulting increments being written as $\mathrm{dC}_{\mathrm{L}}, \mathrm{dC}_{\mathrm{D}}$ and $\mathrm{dC}_{\mathrm{M}}$. Force and moment coefficients were measured using an underfloor balance which had a nominal accuracy of better than $0.05 \%$ full scale deflection for all components. The overall repeatability of balance results was assessed as $\mathrm{dC}_{\mathrm{L}}= \pm 0.0072, \mathrm{dC}_{\mathrm{D}}= \pm 0.0018$ and $\mathrm{dC}_{\mathrm{M}}= \pm 0.0012$. Balance measurements were corrected for blockage $^{14}$ and lift interference ${ }^{19}$. Surface pressure measurements were carried out using the same system described for the two dimensional testing.

The two previously untested damage cases both used a $28 \%$ c diameter hole. The first had an obliquity of $-50^{\circ}$ with zero skew. This obliquity was chosen because it was the largest that could be achieved for this hole size 
without having to cut into the front face of the cavity. The second damage case had $-35^{\circ}$ obliquity and $+55^{\circ}$ skew, with the axis of the hole centres moved so that the upper hole was as close as possible to the leading edge of the panel. This resulted in the axis of the holes being moved forward to 0.43 c. Results for the second case will be presented since the comparisons between experiment and prediction were similar for both cases. In addition, results are only presented for damage at the centre span location. All three span locations were assessed, but differences between wind tunnel and predictions were seen to remain consistent. Pre-requisites for the predictive technique are: 1. Surface static pressure distributions for the undamaged aerofoil.

2. Coefficient increment data for the damaged aerofoil over a range of incidences, This should be obtained for at least two damage cases which cover the range of obliquity angles of interest. The damage size should be reasonably close so that the diameter ratio $\left(\mathrm{F}_{\mathrm{hs}}\right)$ is less than 2 . Data for a $20 \% \mathrm{c}$ hole at $-60^{\circ}$ and $60^{\circ}$ obliquity will be used. The resulting value of $\mathrm{F}_{\mathrm{hs}}$ is 1.4 .

3. Surface static pressure distributions for the undamaged finite aspect ratio wing at the spanwise locations of the damage.

For this paper, all of the above prerequisite data came from wind tunnel measurements, but could equally well have come from computational methods. The following steps are used to provide the predictions:

1. The surface static pressure data for the aerofoil is used to determine the pressure coefficient difference $\left(\mathrm{dC}_{\mathrm{p}}\right)$ at each incidence for each of the damage locations considered for the aerofoil. This is then combined with the coefficient increment data for the aerofoil to produce equivalent plots to Fig. 17.

2. Best fit curves are then placed through the data. In the present paper the best fit curves through the $\pm 60^{\circ}$ obliquity cases are given by equations 4 to 6 .

3. Values of $\mathrm{dC}_{\mathrm{p}}$ for the undamaged finite aspect ratio wing are calculated at each damage location and for each incidence.

4. The coefficient increments for the aerofoil are calculated for each value of $\mathrm{dC}_{\mathrm{p}}$ using the equations for the best fit curves developed in step 2.

5. These increments then need to be converted to the hole size of interest. This is done by using equations 7 and 8 with the appropriate value of $F_{\text {hs }}$ from equation 9. 
6. The increments are currently expressed in terms of the aerofoil geometry. To convert to the geometry of the present finite aspect ratio wing, which is unswept and untapered, requires the use of equations 10 to 12 . The spans and chords are those of the two wind tunnel models which have already been defined.

7. Knowing the relationship between $\mathrm{dC}_{\mathrm{p}}$ and incidence for the finite aspect ratio wing allows the final predictions to be plotted (Fig. 20).

In Fig. 20, the predicted trends are similar to wind tunnel results and this confirms the usefulness of the technique for use in the initial stages of a survivability analysis. Sensitivity studies were carried out by varying the damage cases used to compute the best fit curves in Fig. 17, however these produced little change in the predicted results and the comparisons with wind tunnel data remained essentially the same. For the lift coefficient increment there is a divergence between prediction and experiment at the highest incidences. A similar divergence between finite aspect ratio experiment and prediction was noted during development of the prediction technique for the $20 \% \mathrm{c}$ hole at $-60^{\circ}$ obliquity. The two dimensional experimental data for this damage case (converted to finite aspect ratio geometry by using equations 10 to 12) is compared with finite aspect ratio experimental results for $\mathrm{dC}_{\mathrm{L}}$ in Fig. 21 . The two data points closest to the divergence point at $\mathrm{dC}_{\mathrm{p}}=-0.8$ are for the aerofoil at $\mathrm{dC}_{\mathrm{p}}=-0.791$, which is $4^{\circ}$ of incidence, and $\mathrm{dC}_{\mathrm{p}}=0.768$ for the finite aspect ratio wing at $8^{\circ}$. Flow visualisation of the aerofoil case has been previously shown in Fig. 8b. Flow visualisation for the finite aspect ratio wing is shown in Fig. 22. To retain consistency with previous photographs the image has been rotated so that the leading edge of the model is at the bottom and the right of the photograph is nearest the wing tip. Surface flow visualisation was difficult for the vertically mounted half model because gravity caused liquid that had collected at separation points to flow downwards towards the wing root. This has occurred to the liquid at the centres of the large vortices on the surface of the wing, and for the vortex closest to the tip the liquid has flowed along the separation line between the reverse flow and the expanding jet. The jet on the finite aspect ratio wing is twisted due to the spanwise pressure variation, but based on the findings from Render ${ }^{9}$, this is not expected to produce significant changes in $\mathrm{dC}_{\mathrm{L}}$. Expansion of the jet downstream of the damage hole appears to be more significant for the finite aspect ratio wing, and detailed measurements of the width of the horse shoe vortex indicated that the jet on the finite aspect ratio wing was slightly stronger despite being at a slightly lower $\mathrm{dC}_{\mathrm{p}}$. This difference in jet strength may be due to the relatively small wind tunnel used for the two dimensional testing. It is possible that the closeness of the tunnel walls constrained the 
development of the jet for this extreme obliquity at incidences of $4^{\circ}$ and above. However, it is important to note that none of the other damage cases tested on both the aerofoil and finite aspect ratio wing showed a mismatch.

For the previously untested damage case $d_{D}$ was under predicted over most of the incidence range although the results converge with increasing incidence (Fig. 20b). Part of this under prediction is likely to be due to the value of $\mathrm{F}_{\mathrm{hs}}$ and it is noted from Table 2 that the normalised gradient for the drag coefficient of the $30 \% \mathrm{c}$ hole was 1.36 . This is $10 \%$ less than the value of $\mathrm{F}_{\mathrm{hs}}$, and given the closeness in size of the $28 \% \mathrm{c}$ hole suggests that a similar over estimation of $\mathrm{F}_{\mathrm{hs}}$ may have happened causing the final $\mathrm{dC}_{\mathrm{D}}$ values to be over predicted by around $10 \%$.

The predicted trend with incidence for $\mathrm{dC}_{\mathrm{M}}$ is in line with experiments although absolute values are under predicted (Fig. 20c). Similar discrepancies were seen for all damage cases tested on both the aerofoil and the finite aspect ratio wing, and it is believed that this may well be due to the different mounting methods for the two models. As discussed earlier, compared with NASA data the undamaged aerofoil produced pitching moment increments of smaller magnitude and this was attributed to friction in the pin joints. The half model of the finite aspect ratio wing was mounted directly unto the underfloor balance and this problem did not arise.

\section{Conclusions}

1. The trends in aerodynamic coefficients due to battle damage previously identified by Irwin and Render ${ }^{3}$, are applicable to other aerofoil geometries. These trends are: battle damage increases drag, reduces lift and makes the pitching moment more negative (i.e.: nose down). Up to the onset of stall, these effects became more pronounced as incidence is increased.

2. The addition of negative obliquity (i.e.: the upper surface hole is forward of the lower surface hole) resulted in more significant changes in the aerodynamic coefficients. Positive obliquity reduced the magnitude of changes in the aerodynamic coefficients.

3. For all damage cases with a given hole size, there is a broad trend when coefficient increments are plotted against the difference in pressure coefficients between the upper and lower surfaces on an undamaged aerofoil at the damage locations. Best fit curves through the data can be used as the basis of a method to predict the effects of 
battle damage on a finite aspect ratio wing. This technique has been demonstrated by comparing predictions with wind tunnel results for two previously untested cases.

\section{Acknowledgments}

This work has been supported by BAE Systems as part of their contribution to the European BaToLUS (Battle Damage Tolerance to Lightweight UAV Structures) project. The enthusiastic monitoring of the project by Dr. Andrew Irwin and Mark Lucking has been greatly appreciated by the authors. The help and expertise of Rob Hunter and Stacey Prentice in manufacturing the wind tunnel models is also appreciated.

\section{References}

1. Saeedi, M., Ajalli, F. and Mani, M. “A Comprehensive Numerical Study of Battle Damage and Repairs upon the Aerodynamic Characteristics of an Aerofoil”, The Aeronautical Journal, Vol 114, No 1158, pp 469-484, 2010.

2. Yang, Z., Samad-Suhaeb, M., Render, P.M. “Computational Study of a Battle Damaged Finite Aspect Ratio Wing”, 30th AIAA Applied Aerodynamics Conference, New Orleans, USA, pp 983-991, 2012.

3. Irwin, A.J. and Render, P.M. "The Influence of Mid-chord Battle Damage on the Aerodynamic Characteristics of Two-dimensional Wings”, The Aeronautical Journal, Vol.104, No. 1033, pp153-161, March 2000.

4. Andreopoulos, J. and Rodi, W. "Experimental Investigation of jets in a crossflow”, Journal of Fluid Mechanics, Vol 138, 1984.

5. Mahesh, K. "The interaction of Jets with Crossflow”, Annual Review of Fluid Mechanics, Vol 45, pp 379-407, 2013. DODI 10.1146/annurev-fluid-120710-101115

6. Irwin, A.J. and Render, P.M. "The Influence of Internal Structure on the Aerodynamic Characteristics of Battle Damaged Wings”, 14th AIAA Applied Aerodynamic Conference Proceedings, Paper 1996-2395, 1996.

7. Render, P.M., de Silva, S., Walton A.J. and Mahmoud, M. "Experimental Investigation into the Aerodynamic Effects of Battle Damaged Airfoils", AIAA Journal of Aircraft, Vol 44, No 2, pp 539-549, 2007. DOI $10.2514 / 1.24144$

8. Robinson, K.W. and Leishman, J.G. "Effects of Ballistic Damage on the Aerodynamics of Helicopter Rotor Airfoils”, AIAA Journal of Aircraft, Vol 33, No 5, pp 695-703, 1998.

9. Render, P.M., Samad-Suhaeb, M., Yang, Z. and Mani, M. “Aerodynamics of Battle-Damaged Finite-Aspect Ratio Wings”, AIAA Journal of Aircraft, Vol 46, No 3, pp 997-1004, 2009. DOI: 102514/1.39839. 
10. Render, P.M. and Pickhaver, T.W. "The Influence of Hole Orientation on the Aerodynamics of a Battle Damaged Wing”, 30 ${ }^{\text {th }}$ AIAA Applied Aerodynamics Conference Proceedings, pp 800-813, 2012.

11. Pickhaver, T.W. and Render, P.M. “A Technique to Predict the Aerodynamic Losses of Battle Damaged Wings”, $28^{\text {th }}$ Congress of the International Council of the Aeronautical Sciences Proceedings, Vol 2, pp 914 -928, 2012.

12. McGhee, R.J. and Beasley, W.D. "Wind-tunnel results for a modified 17-percent-thick low-speed airfoil section”, NASA Technical Paper 1919, 1981.

13. "Lift Interference and Blockage Corrections for Two-Dimensional Subsonic Flow in Ventilated and Closed Wind Tunnels”, Engineering Sciences Data Unit, Data sheet ESDU 76028, 1995.

14. Garner, H.C., Rogers, E.W.E., Acum, W.E.A. and Maskell, E.C. "Subsonic Wind Tunnel Corrections", Technical Report AGARDograph 109, AGARD, October 1966.

15. Compton, D.A. and Johnston, J.P. "Streamwise Vortex Production by Pitched and Skewed Jets in a Turbulent Boundary Layer", AIAA Journal, Vol. 30, No. 3, pp. 640-647, 1992.

16. Milanovic, I.M. and Zaman, K.B.M.Q. "Fluid Dynamics of Highly Pitched and Yawed Jets in Crossflow”, AIAA Journal, Vol 42, No 5, pp 874-882, 2004.

17. Irwin, A.J. “Investigation into the Aerodynamic Effects of Simulated Battle Damage to a Wing”, PhD Thesis, Loughborough University, 1999.

18. Pickhaver, T. W. "Prediction and Validation of the Aerodynamic Effects of Simulated Battle Damage on Aircraft Wings”, PhD Thesis, Loughborough University, 2013.

19. “Upwash Interference for Wings in Solid-liner Wind Tunnels using Subsonic Linearised-Theory”, Engineering Sciences Data Unit, Data sheet ESDU 95014, 1995.

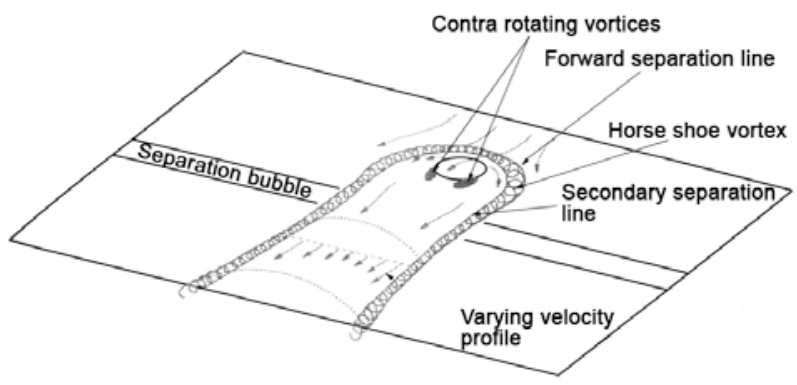

Figure 1. Weak jet sketch. 


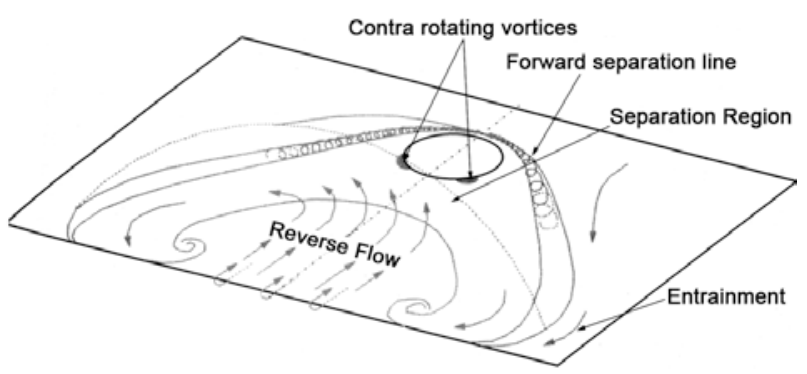

Figure 2. Strong jet sketch.

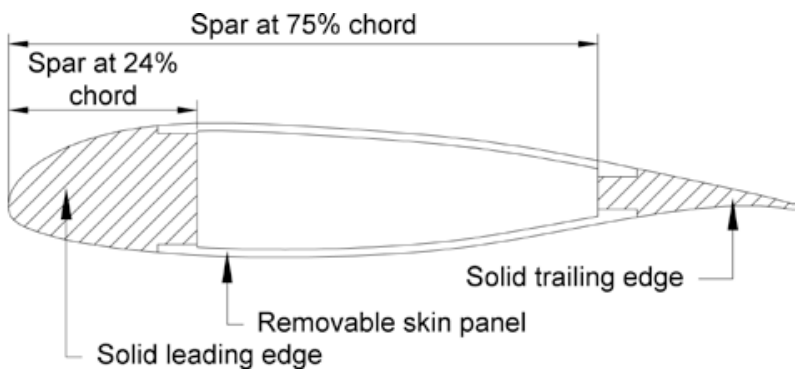

Figure 3. Cross-section of aerofoil model

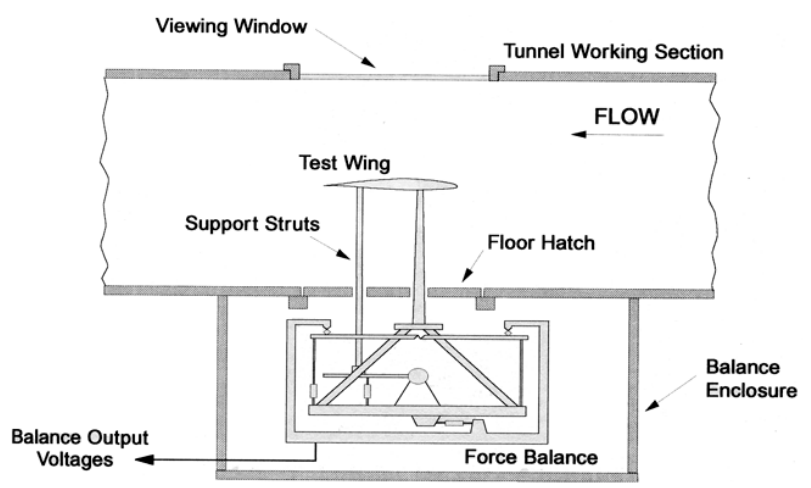

Figure 4. Experimental arrangement in wind tunnel. 


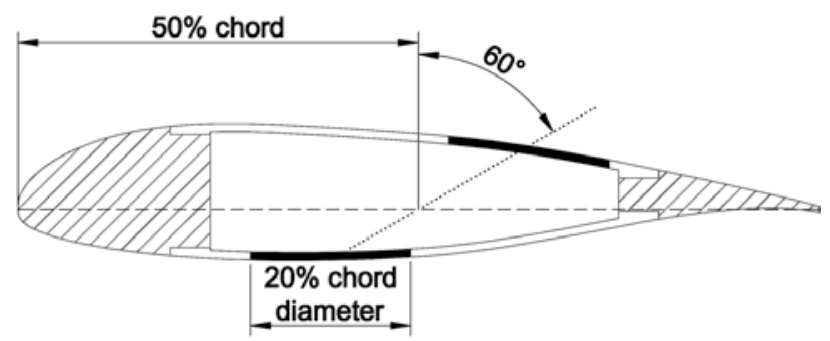

Figure 5. Sketch showing definition of $+60^{\circ}$ obliquity.

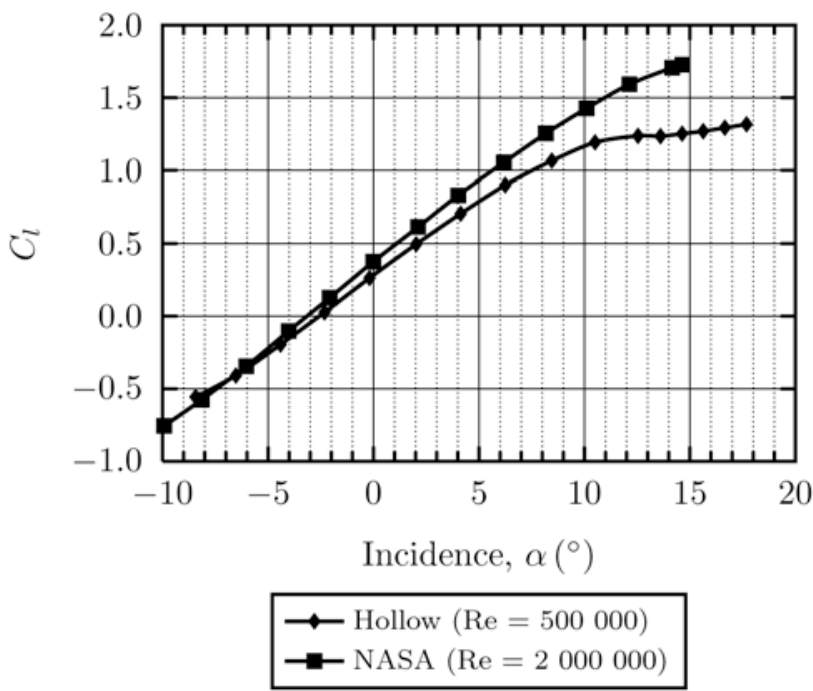

a) Lift

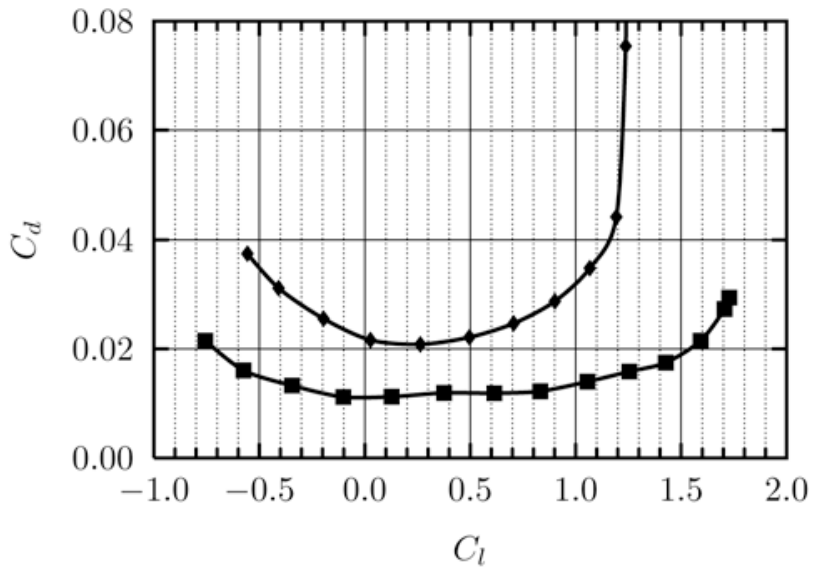

$\longrightarrow$ Hollow $(\mathrm{Re}=500000)$

$\rightarrow$ NASA $(\operatorname{Re}=2000000)$

b) Drag 


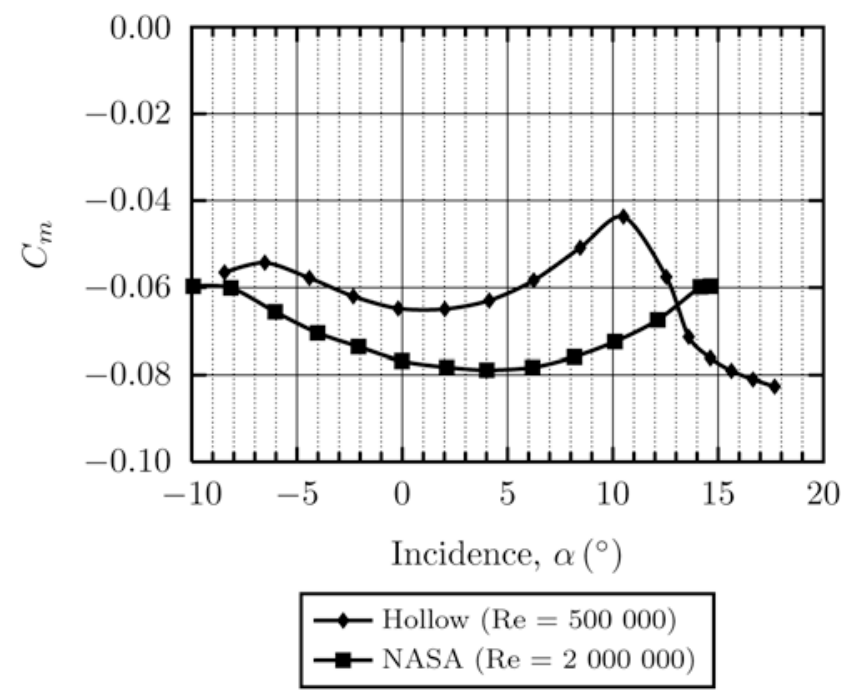

c) Pitching Moment

Figure 6. Comparison of undamaged model and NASA data ${ }^{12}$

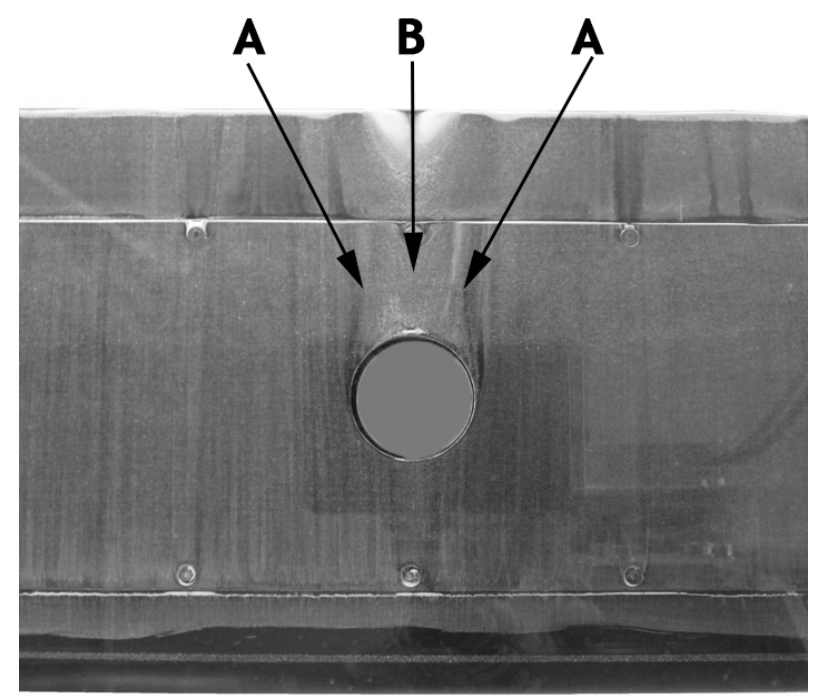

a) $0^{\circ}$ Incidence 


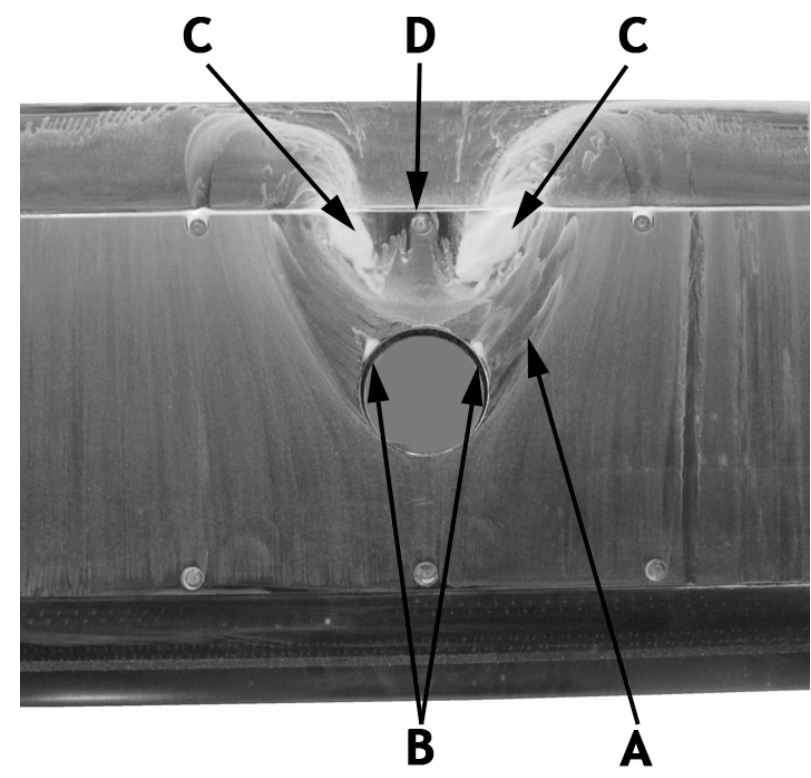

b) $4^{\circ}$ Incidence

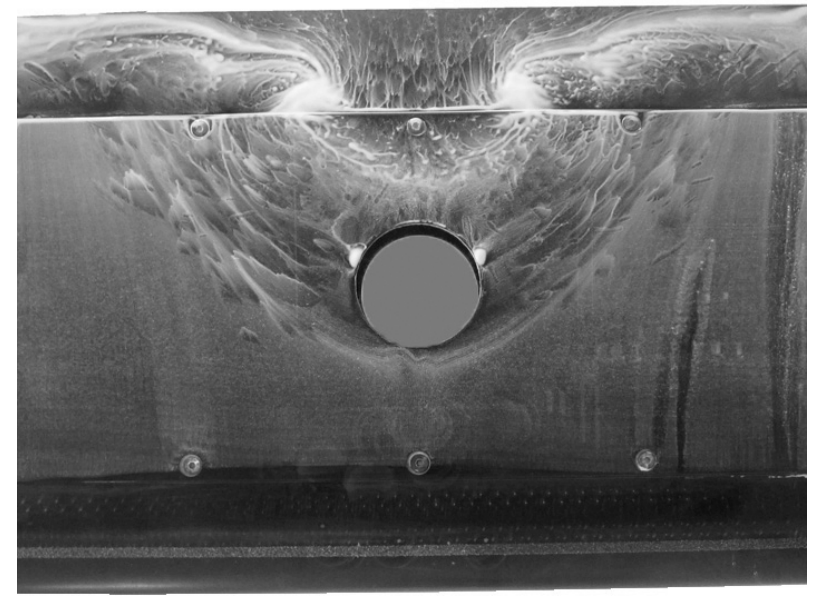

c) $8^{\circ}$ Incidence

Figure 7. Flow visualisation on upper surface of model for straight through damage. 


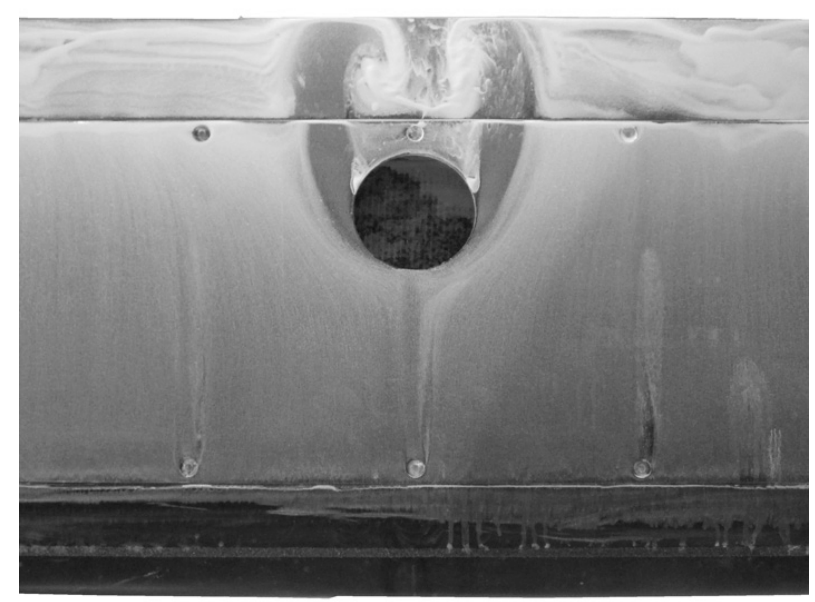

a) Obliquity $=+60^{\circ}$

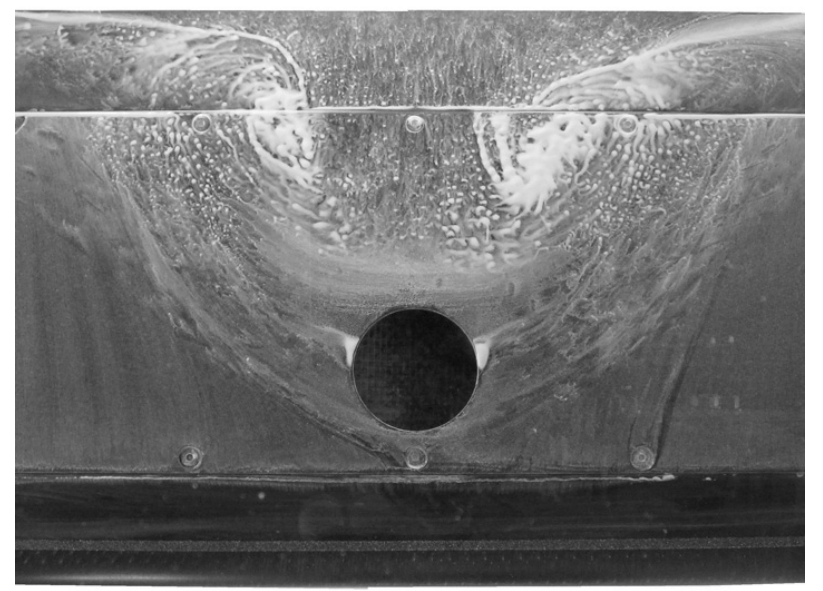

b) Obliquity $=-60^{\circ}$

Figure 8. Flow visualisation on upper surface for different obliquity angles. $20 \%$ c Damage. Incidence $=\mathbf{8}^{\circ}$. 


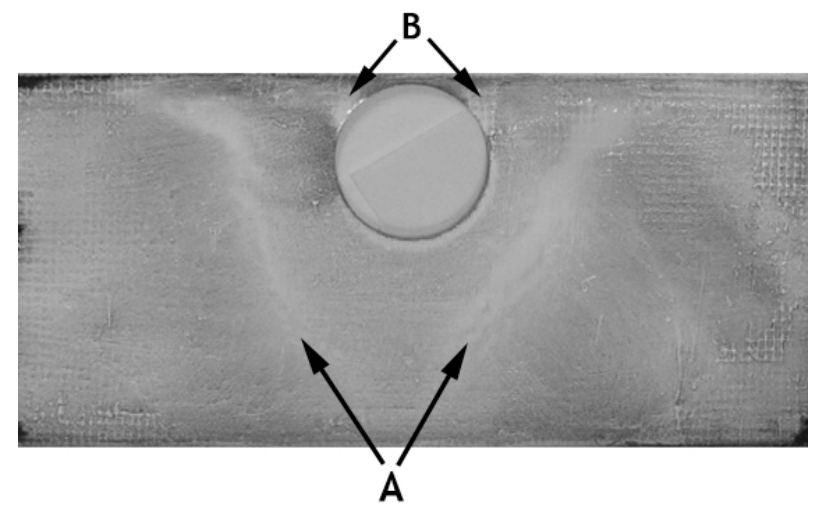

a) Lower panel

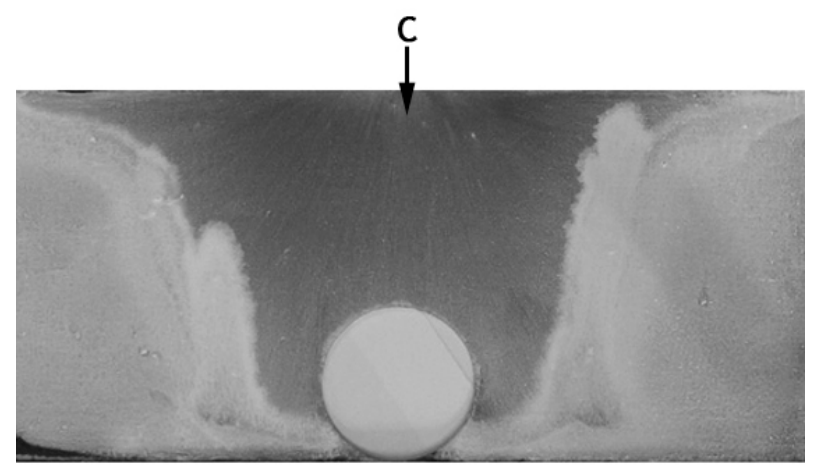

b) Upper panel

Figure 9. Internal flow visualisation. $20 \%$ c damage. $-60^{\circ}$ obliquity. $8^{\circ}$ incidence. (Front of each panel at the bottom of the photograph.) 


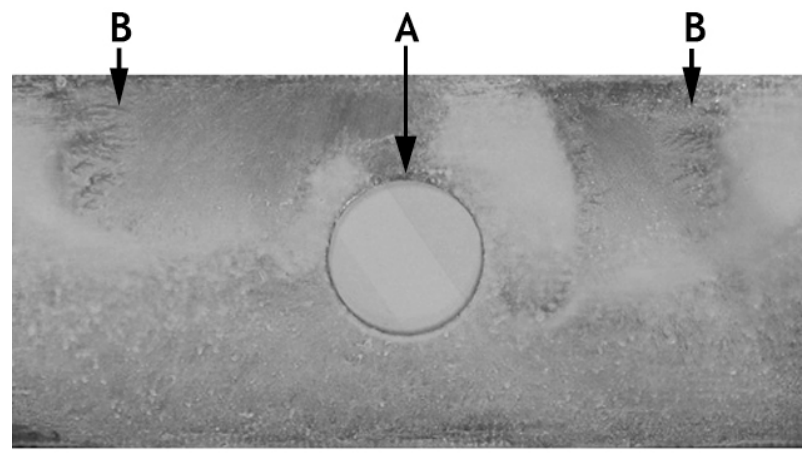

a) Lower panel

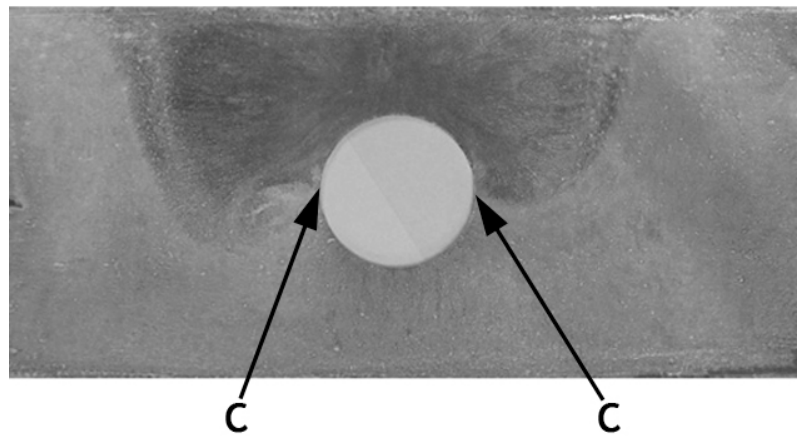

b) Upper panel

Figure 10. Internal flow visualisation. 20\%c straight through damage. $8^{\circ}$ incidence. (Front of each panel at the bottom of the photograph.) 


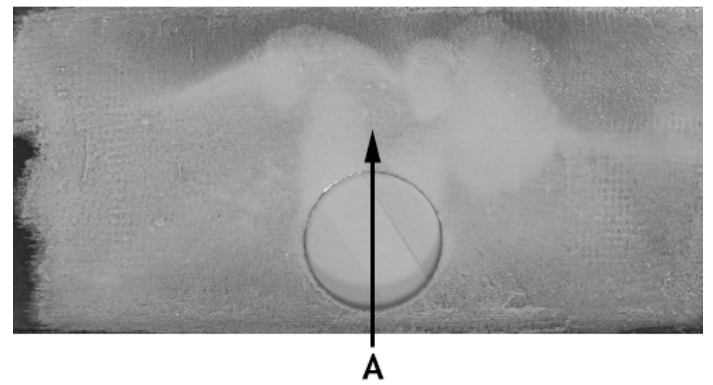

a) Lower panel

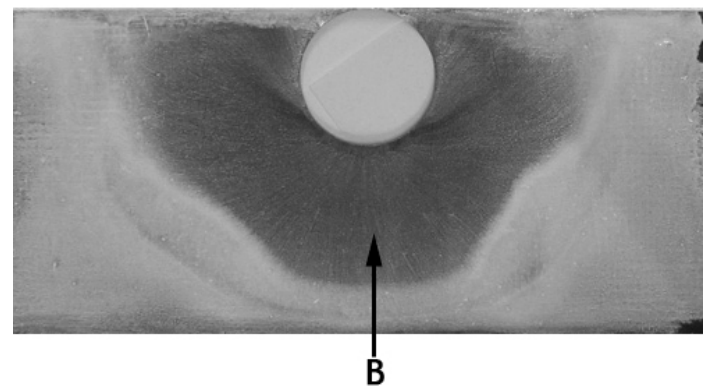

b) Upper panel

Figure 11. Internal flow visualisation. $20 \%$ c damage. $+60^{\circ}$ obliquity. $8^{\circ}$ incidence. (Front of each panel at the bottom of the photograph.) 

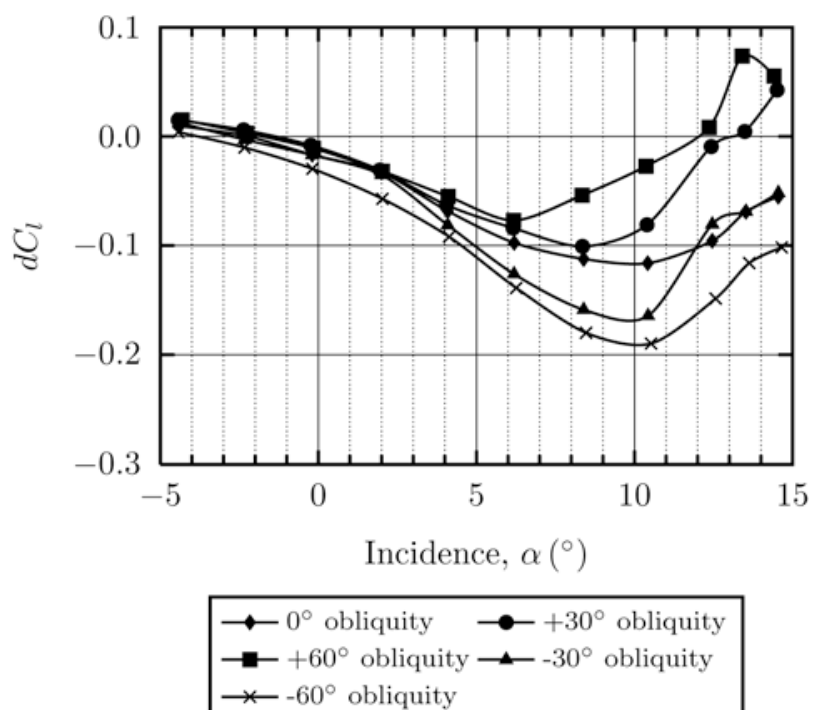

a) Lift

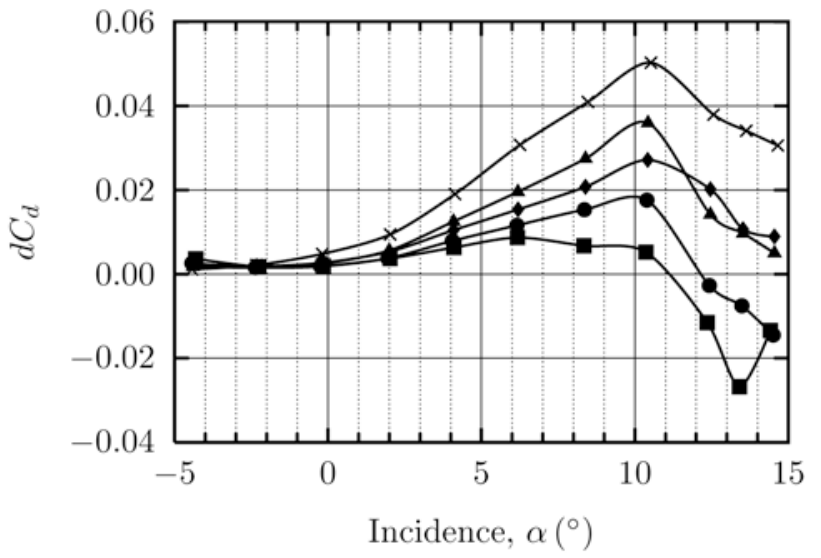

$$
\begin{aligned}
& \longrightarrow-0^{\circ} \text { obliquity } \longrightarrow-30^{\circ} \text { obliquity } \\
& \longrightarrow-+60^{\circ} \text { obliquity } \longrightarrow-30^{\circ} \text { obliquity } \\
& \rightarrow \bullet-60^{\circ} \text { obliquity }
\end{aligned}
$$

b) Drag 


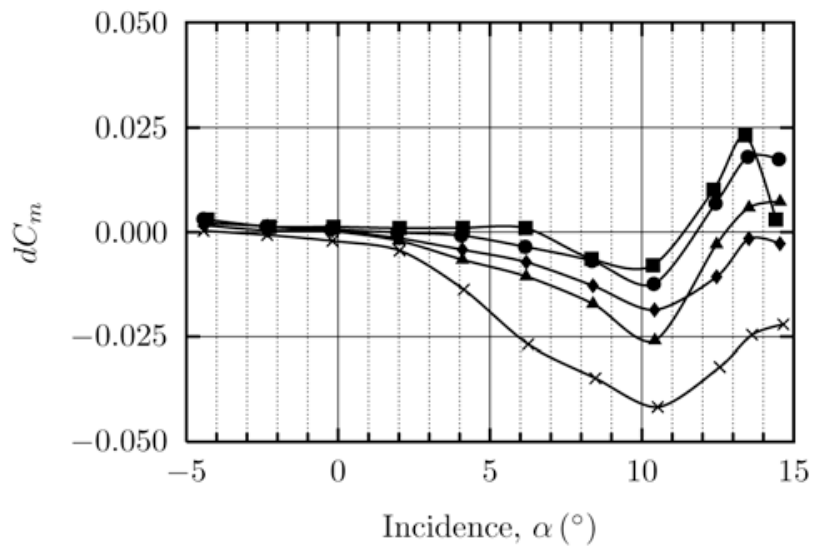

$$
\begin{aligned}
& \longrightarrow 0^{\circ} \text { obliquity } \longrightarrow+30^{\circ} \text { obliquity } \\
& \longrightarrow-+60^{\circ} \text { obliquity } \longrightarrow-30^{\circ} \text { obliquity } \\
& \rightarrow-60^{\circ} \text { obliquity }
\end{aligned}
$$

c) Pitching Moment

Figure 12. Coefficient increments for different obliquity angles. 


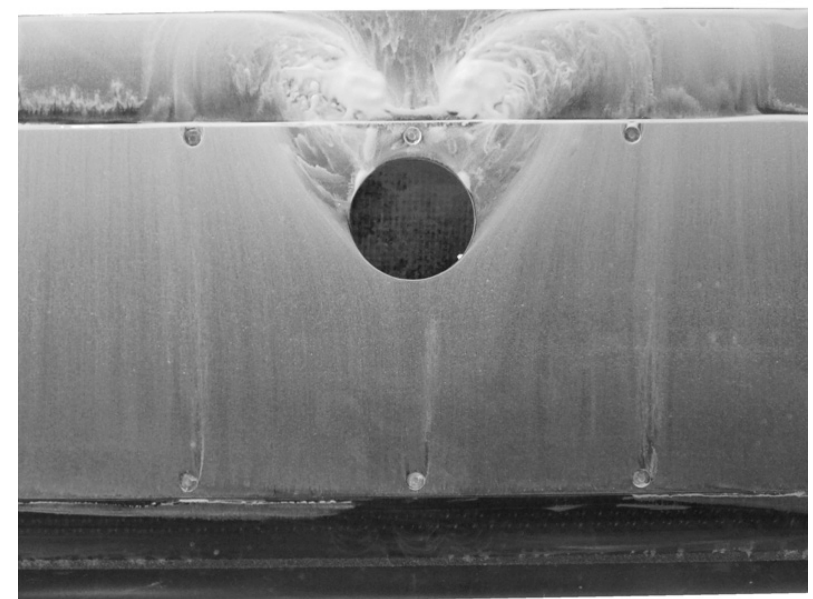

a) Incidence $4^{\circ}$

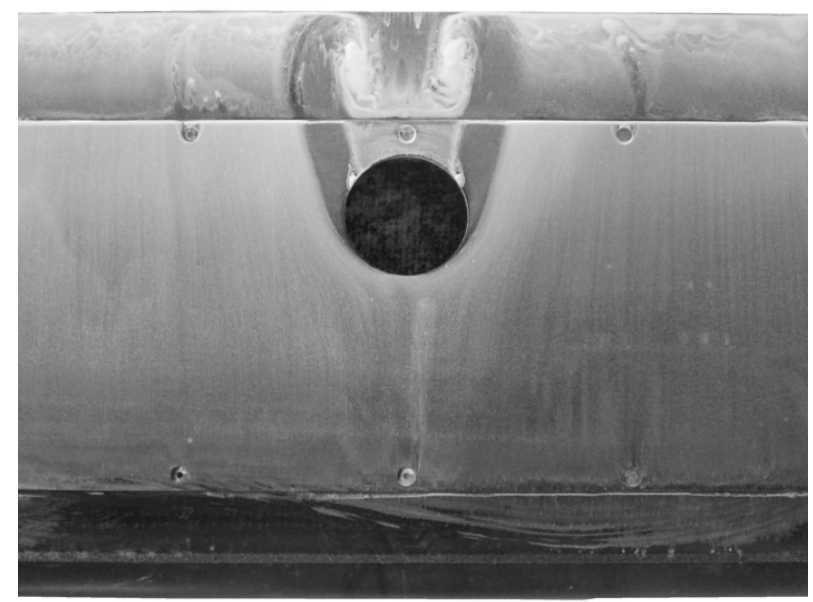

b) Incidence $6^{\circ}$

Figure 13. Flow visualisation on upper surface for $+60^{\circ}$ obliquity. 


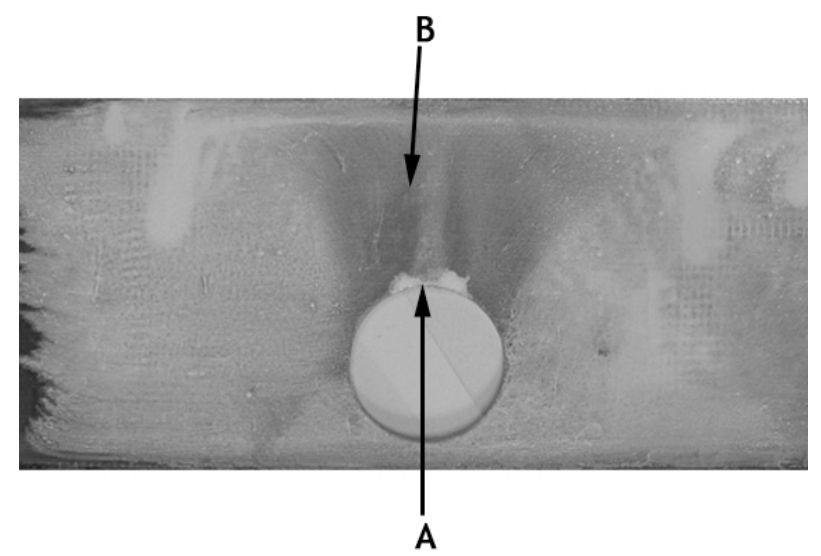

a) Lower panel

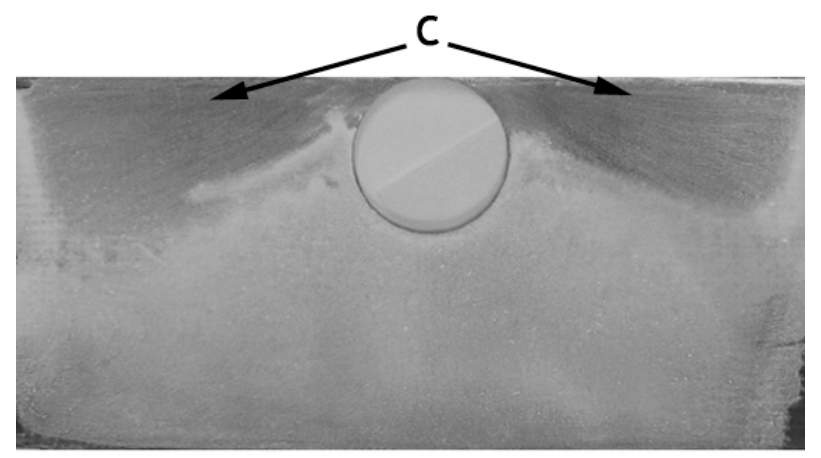

b) Upper panel

Figure 14. Internal flow visualisation. $20 \%$ c damage. $+60^{\circ}$ obliquity. $4^{\circ}$ incidence. (Front of each panel at the bottom of the photograph.) 


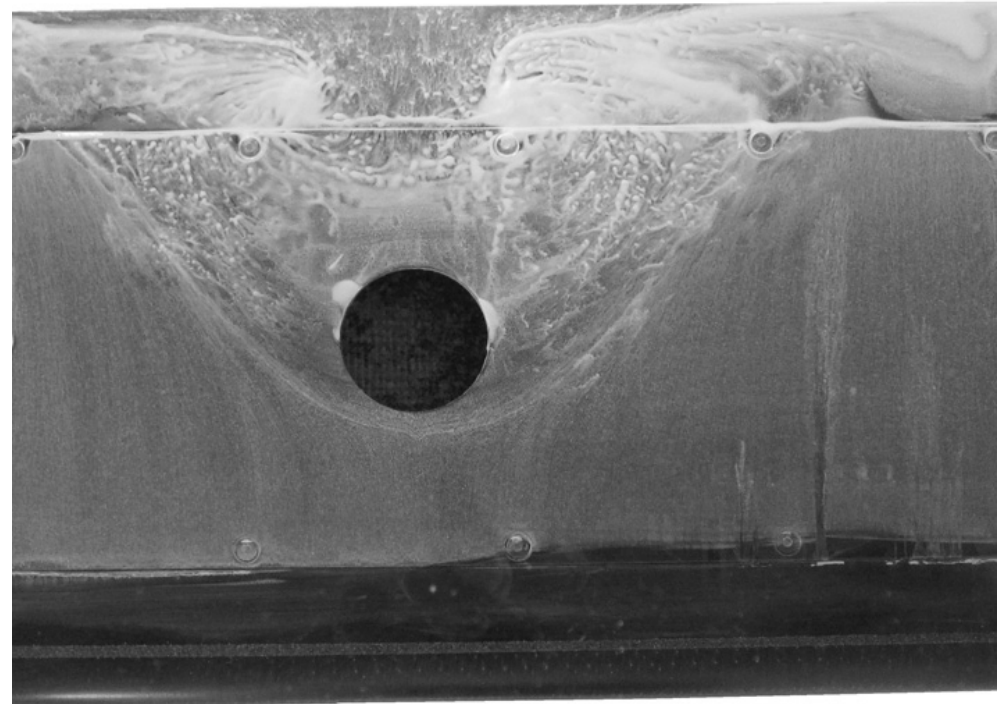

Figure 15. Flow visualisation on upper surface for $60^{\circ}$ skew. Incidence $=8^{\circ}$. 


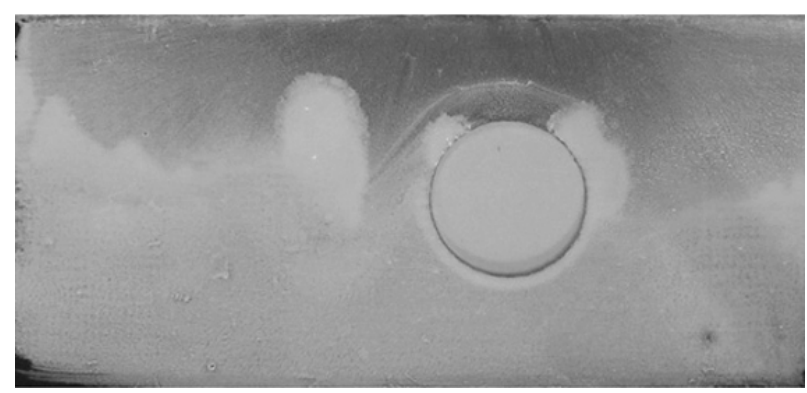

a) Lower panel

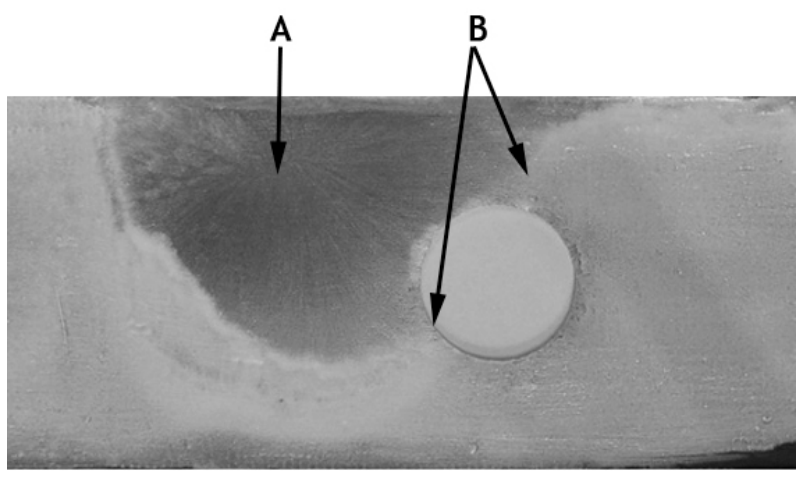

b) Upper panel

Figure 16. Internal flow visualisation. $20 \% \mathrm{c}$ damage. $60^{\circ}$ skew. $8^{\circ}$ incidence. (Front of each panel at the bottom of the photograph.) 


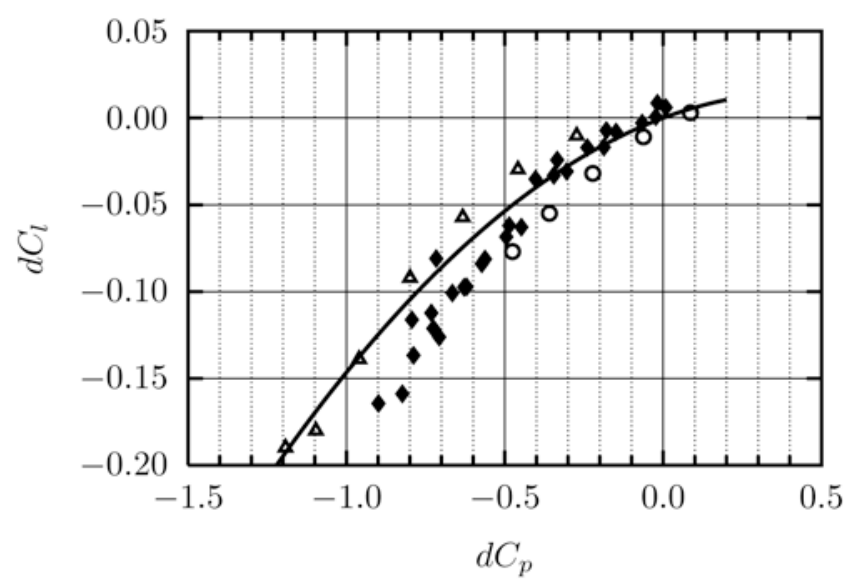

- Experimental data - Line of best fit

$\Delta-60^{\circ}$ obliquity $\quad \circ+60^{\circ}$ obliquity

a) Lift
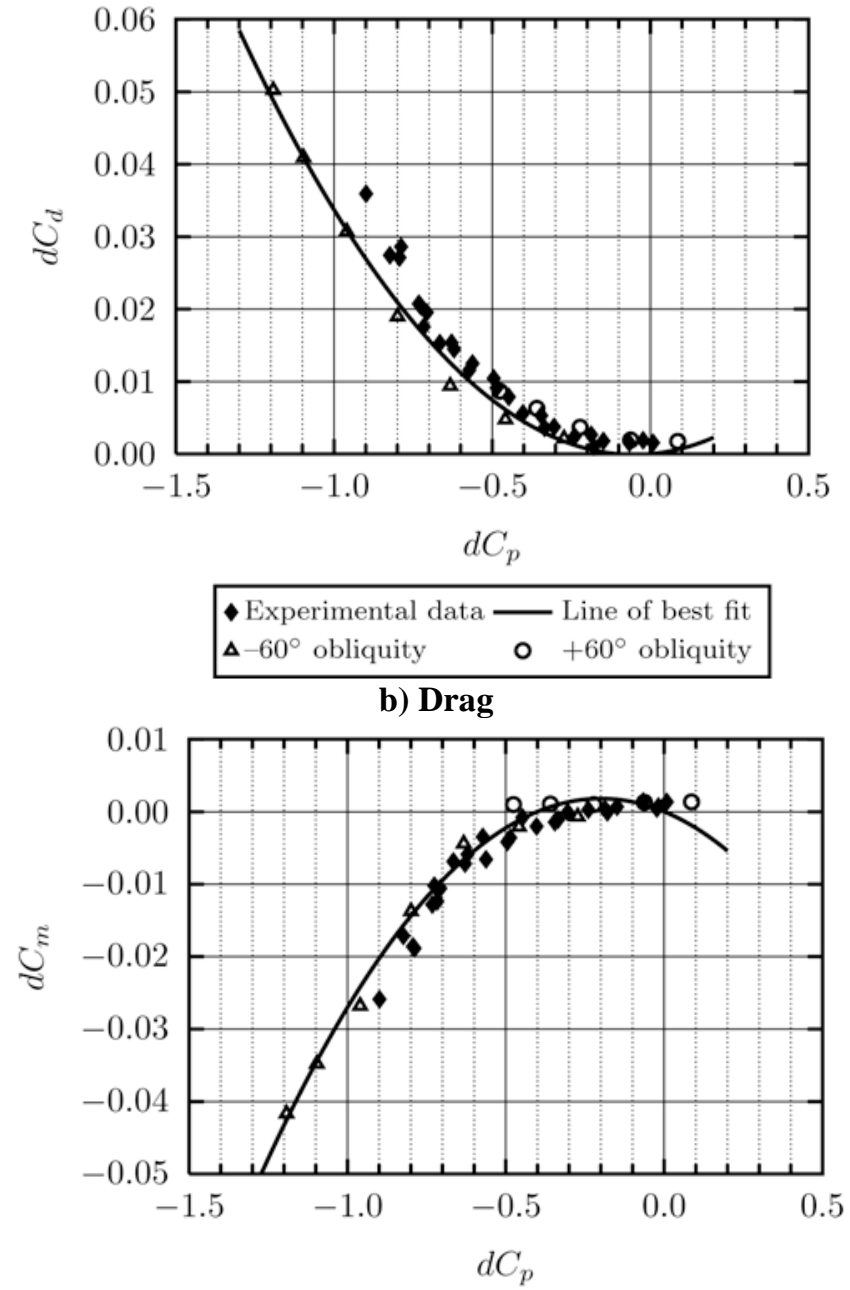

- Experimental data — Line of best fit

$\Delta-60^{\circ}$ obliquity $\quad \circ+60^{\circ}$ obliquity

c) Pitching moment

Figure 17. Coefficient increments for $20 \%$ c diameter plotted against pressure coefficient difference. 

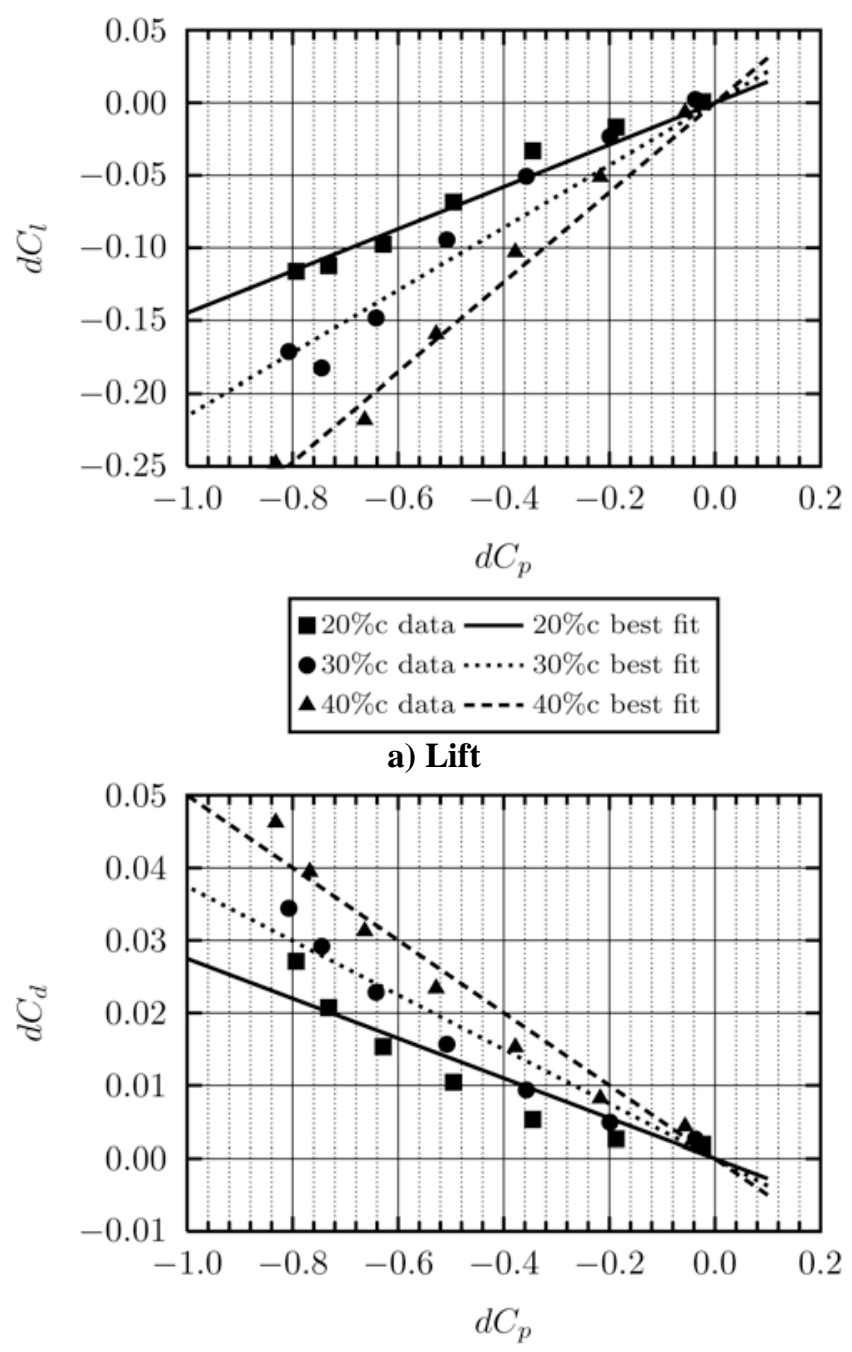

- $20 \%$ c data $-20 \%$ c best fit

- $30 \%$ c data $\cdots \cdots 30 \%$ c best fit

A $40 \%$ c data - - $40 \%$ c best fit

\section{b) Drag}




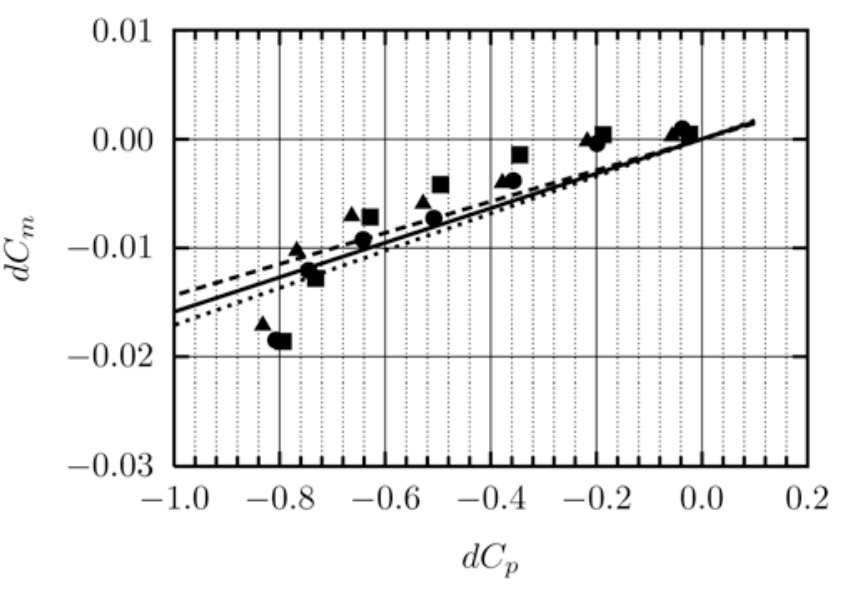

- $20 \%$ c data $-20 \%$ c best fit

- $30 \%$ c data $\cdots . .30 \%$ c best fit

A $40 \%$ c data - - $40 \%$ c best fit

c) Pitching moment

Figure 18. The influence of hole size on coefficient increments.

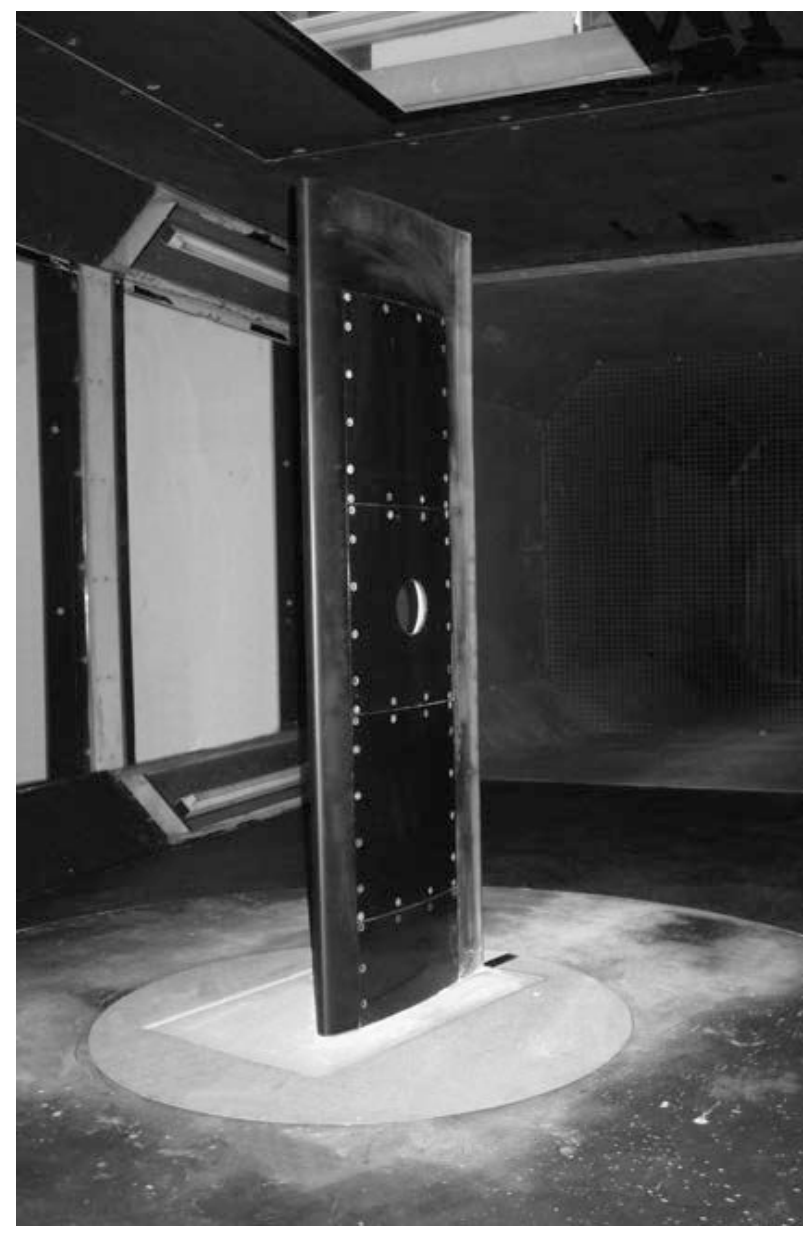

Figure 19. Finite aspect ratio wing half model wind tunnel arrangement. 


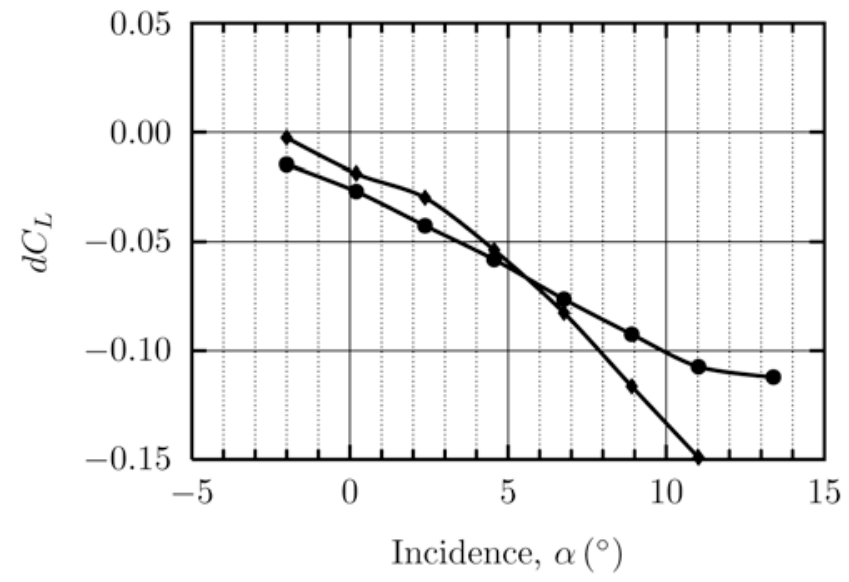

$\multimap$ 3D experimental $\bullet$ Prediction

a) Lift

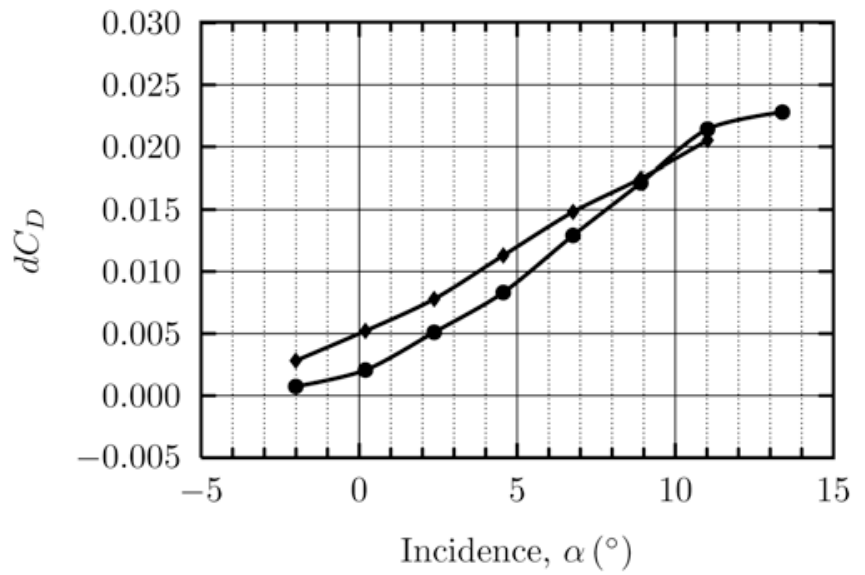

$\multimap$ 3D experimental $\bullet$ Prediction

b) Drag

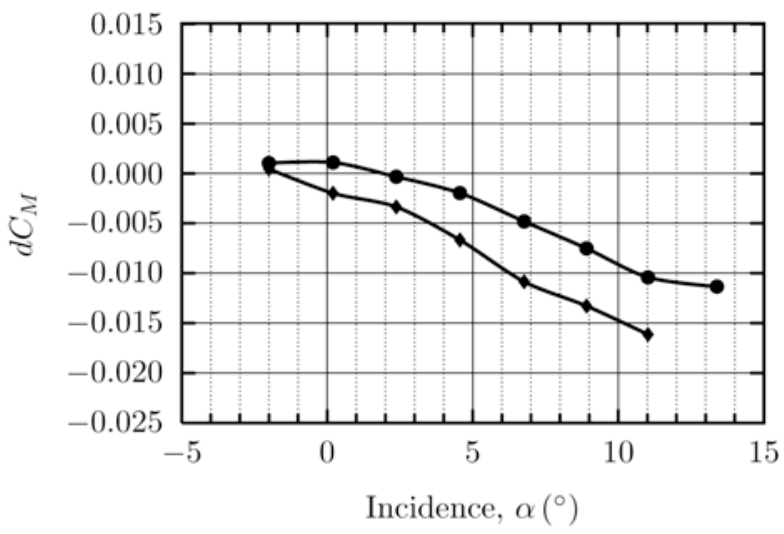

$\multimap$ 3D experimental $\multimap-$ Prediction

c) Pitching moment 
Figure 20. Predictions for previously untested damage case on finite aspect ratio wing. $28 \%$ c diameter. $-35^{\circ}$ obliquity. $55^{\circ}$ skew

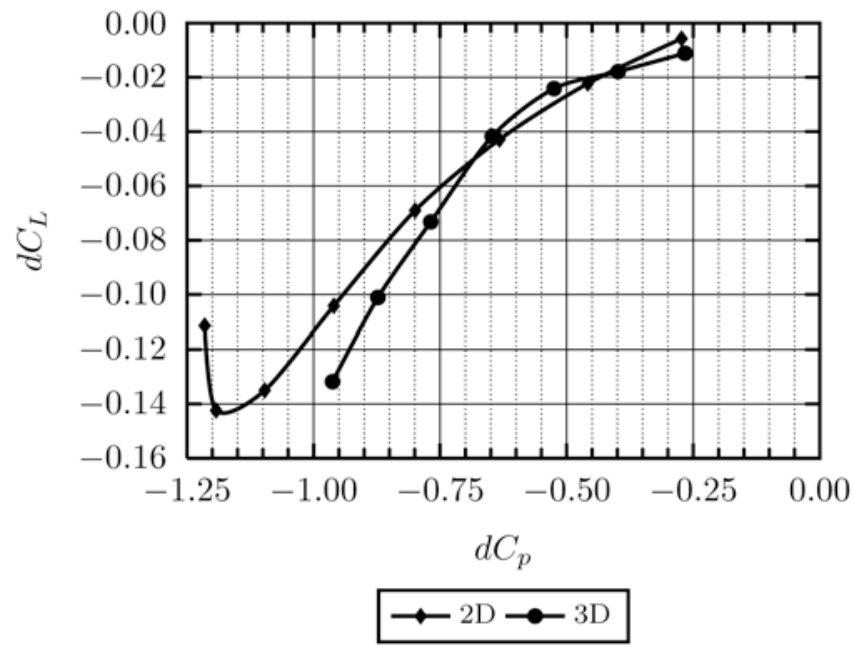

Figure 21. Experimental lift coefficient increments for aerofoil (2D) and finite aspect ratio (3D) models. $60^{\circ}$ obliquity.

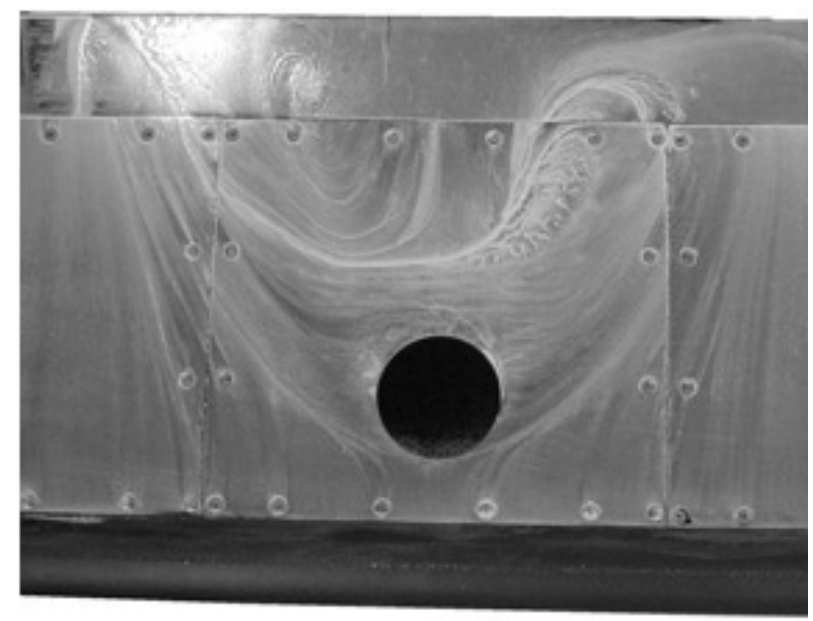

Figure 22. Flow visualisation of finite aspect ratio wing. $8^{\circ}$ incidence. $-60^{\circ}$ obliquity. Centre span.

\begin{tabular}{cccccc}
\hline \hline & & & Obliquity & \\
Skew & $\mathbf{- 6 0}^{\mathbf{0}}$ & $-\mathbf{3 0}^{\mathbf{0}}$ & $\mathbf{0}^{\mathbf{0}}$ & $\mathbf{3 0}^{\mathbf{0}}$ & $\mathbf{6 0}^{\mathbf{0}}$ \\
\hline $\mathbf{0}^{\mathbf{0}}$ & $20 \% \mathrm{c}$ & $20 \% \mathrm{c}$ & $20 \% \mathrm{c}$ & $20 \% \mathrm{c}$ & $20 \% \mathrm{c}$ \\
& & & $30 \% \mathrm{c}$ & & \\
& & & $40 \% \mathrm{c}$ & & \\
$\mathbf{3 0}^{\mathbf{0}}$ & & $20 \% \mathrm{c}$ & $20 \% \mathrm{c}$ & $20 \% \mathrm{c}$ & \\
$\mathbf{4 5}^{\mathbf{0}}$ & $20 \% \mathrm{c}$ & & $20 \% \mathrm{c}$ & & $20 \% \mathrm{c}$ \\
& & $40 \% \mathrm{c}$ & & \\
$\mathbf{6 0}^{\mathbf{0}}$ & & $20 \% \mathrm{c}$ & & \\
\hline \hline
\end{tabular}

Table 1 


\begin{tabular}{lccc}
\hline \hline $\begin{array}{c}\text { Hole } \\
\text { Diameter }\end{array}$ & $\mathbf{d C}_{\mathbf{l}} / \mathbf{d C}_{\mathbf{p}}$ Gradient & $\mathbf{d C}_{\mathbf{d}} / \mathbf{d C}_{\mathbf{p}}$ Gradient & $\begin{array}{c}\text { Normalised } \\
\text { Diameter }\end{array}$ \\
\hline $30 \% \mathrm{c}$ & 1.49 & 1.36 & 1.5 \\
$40 \% \mathrm{c}$ & 2.13 & 1.82 & 2 \\
\hline \hline
\end{tabular}

Table 2 